\title{
MEBN-RM: A Mapping between Multi-Entity Bayesian Network and Relational Model
}

\author{
Cheol Young Park ${ }^{1, *(D)}$ and Kathryn Blackmond Laskey ${ }^{2}$ \\ 1 The C4I and Cyber Center, George Mason University, Fairfax, VA 22030, USA \\ 2 The Department of Systems Engineering and Operations Research, George Mason University, \\ Fairfax, VA 22030, USA; klaskey@gmu.edu \\ * Correspondence: cparkf@gmu.edu
}

Received: 12 March 2019; Accepted: 23 April 2019; Published: 26 April 2019

\begin{abstract}
Multi-Entity Bayesian Network (MEBN) is a knowledge representation formalism combining Bayesian Networks (BNs) with First-Order Logic (FOL). MEBN has sufficient expressive power for general-purpose knowledge representation and reasoning, and is the logical basis of Probabilistic Web Ontology Language (PR-OWL), a representation language for probabilistic ontologies. Developing an MEBN model to support a given application is a challenge, requiring definition of entities, relationships, random variables, conditional dependence relationships, and probability distributions. When available, data can be invaluable both to improve performance and to streamline development. By far the most common format for available data is the relational database (RDB). Relational databases describe and organize data according to the Relational Model (RM). Developing an MEBN model from data stored in an RDB therefore requires mapping between the two formalisms. This paper presents MEBN-RM, a set of mapping rules between key elements of MEBN and RM. We identify links between the two languages (RM and MEBN) and define four levels of mapping from elements of RM to elements of MEBN. These definitions are implemented in the MEBN-RM algorithm, which converts a relational schema in RM to a partial MEBN model. Through this research, the software has been released as an MEBN-RM open-source software tool. The method is illustrated through two example use cases using MEBN-RM to develop MEBN models: a Critical Infrastructure Defense System and a Smart Manufacturing System. Both systems are proof-of-concept systems used for situation awareness, where data coming from various sensors are stored in RDBs and converted into MEBN models through the MEBN-RM algorithm. In these use cases, we evaluate the performance of the MEBN-RM algorithm in terms of mapping speed and quality to show its efficiency in MEBN modeling.
\end{abstract}

Keywords: mapping model; probabilistic graphical model; Multi-Entity Bayesian Networks; Relational Model; semantic web; Probabilistic Web Ontology Language; Statistical Relational Learning; MEBN modeling; MEBN machine learning

\section{Introduction}

Statistical Relational Learning (SRL) deals with representation and reasoning methods for uncertain and complex situations by combining probabilistic models (e.g., BNs and Markov Networks) and relational structures (e.g., FOL and RM) [1]. The expressive power of SRL enables us to represent real-world situations characterized by uncertainty and complexity. For this reason, it has been used in several domains (e.g., information fusion [2], video analysis [3,4], and bioinformatics [5]). Several formalisms embodying probabilistic models with relational structures have been proposed in recent decades, such as Probabilistic-Logic Programming [6], Programming in Statistical Modeling [7], Probabilistic RMs [8], Relational BNs [9], Relational Markov Networks [10], Bayesian 
LOGic [11], Markov Logic Networks [12], Conditional Random Fields for Logical Sequences [13], Bayesian Logic Programming [14], FACTORIE: Probabilistic Programming [15], and Probabilistic Conditional Logic [16].

Multi-Entity Bayesian Network (MEBN) belongs to the formalisms in SRL. MEBN is a knowledge representation language based on Bayesian Networks (BN) [17] and First-Order Logic (FOL). MEBN is sufficiently expressive for general-purpose knowledge representation and reasoning in an uncertain and complex world. Because MEBN is flexible enough to represent a variety of complex and uncertain situations, it has been applied to systems for Predictive Situation Awareness (PSAW), the problem of understanding and predicting aspects of a temporally evolving situation [18-27]. In a recent review of knowledge representation formalisms, Golestan et al. [28] recommended MEBN as having the most comprehensive coverage of features needed to represent complex problems among several Artificial Intelligence (AI) models including statistical RMs. The reviewed formalisms included MEBN, Hidden Markov Models [29], Artificial Neural Networks [30], BNs [17], Support Vector Machine [31], Fuzzy BNs [32], Dynamic BNs [33], Probabilistic RMs [1], and Markov Logic Networks [12]. Also, MEBN has been applied to a wide variety of domains [34].

Construction of relational structures (e.g., FOL and RM) for MEBN is an active research topic. Probabilistic Web Ontology Language (PR-OWL) [35] extends the Web Ontology Language (OWL) to represent uncertainty. A domain ontology, typically represented in a relational language, provides common semantics for expressing information about entities and relationships in a domain. PR-OWL is an upper ontology, written in OWL, that uses MEBN to express uncertainty about entities and relationships in an OWL ontology. PR-OWL 2 extends PR-OWL to provide better integration with OWL [36]. Carvalho et al. [37] provides a methodology for developing MEBN theories expressed as PR-OWL ontologies. PR-OWL, a semantic web language associated with probability, can be used as a common language for the Internet of Things (IoT) [38] and the Web of Things (WoT) [39]. IoT is a network composed of a group of physical systems that interact with each other over the Internet using sensors, actuators, and computing devices. WoT is an extension of IoT by including virtual systems (i.e., the Internet Web) to deliver services to stakeholders. Sekkal et al. [40] used PR-OWL as a representation formalism specifically representing watering system situations in a WoT architecture. The paper showed that the sensor data obtained through IoT can be used to predict the humidity of the ground to support decision making of watering.

This paper focuses on the RM, from which MEBN theories can be partially constructed. The Relational Model (RM) [41-43] is the most popular database model. While non-relational databases, called NoSQL, are receiving increasing attention [44], our focus in this work is on RM because so much of the available data is stored in relational databases. A Relational Database (RDB) uses RM to describe and organize data. An RDB stores data in the form of multiple relations. A relation is composed of a relation schema and a relation instance. The relation schema represents a class of entities and its attributes. A relational database schema or relational schema is a collection of relation schemas. An MEBN model, called an MTheory, consists of a set of MFrags. An MFrag is composed of Context nodes, Input nodes, Resident nodes, a fragment graph, and a set of Local Distributions.

To construct an MTheory from an RDB, we need a way to map from relations to MFrags. In this paper, we introduce a mapping between a relational schema and a partial MTheory. This mapping is called MEBN-RM mapping (or MEBN-RM). MEBN-RM contains four levels of mapping from elements of a relational database to elements of an MTheory. The first level maps a relation schema to an entity in an MTheory. The second level maps attributes of a relation schema to resident nodes of an MFrag. The third maps a relation schema to an MFrag is defined. The fourth level maps a relational database to an MTheory. Furthermore, MEBN-RM forms the basis for a MEBN-RM mapping algorithm takes a relational database as input and produces a partial MTheory as output.

The main contribution of this paper is to introduce the set of mapping definitions and the MEBN-RM mapping algorithm, which can be the basis for developing MEBN theories in manual approaches (e.g., [37]) or automatic approaches (e.g., [45-47]). In addition, we present an 
MEBN-RM open-source software tool that is implemented in the Java programming language and performs MEBN-RM.

The remainder of the paper is organized as follows. Section 2 provides background knowledge of MEBN and RM. Section 3 defines MEBN-RM and presents the mapping algorithm. Section 4 introduces the MEBN-RM open-source software tool and an experiment for MEBN-RM algorithm performance in terms of the mapping time and accuracy. Section 5 presents two use cases in which the tool is applied to construct a partial MTheory. The final section presents conclusions and future research directions.

\section{Background}

In this section, we describe MEBN, a graphical representation for MEBN, and a script form of MEBN. Then, RM is presented briefly along with examples.

\subsection{Multi-Entity Bayesian Network}

MEBN [48] allows compact representation of repeated structure in a joint distribution on a set of random variables. In MEBN, random variables are defined as templates that can be repeatedly instantiated to construct probabilistic models with repeated structure. MEBN represents domain knowledge using an MTheory, which consists of a collection of MFrags (see Figure 1). An MFrag is a fragment of a graphical model that is a template for probabilistic relationships among instances of its random variables. Random variables (RVs) may contain ordinary variables, which can be instantiated for different domain entities. We can think of an MFrag as a class which can generate instances of BN fragments. These can then be assembled into a Bayesian network, called a situation-specific Bayesian Network (SSBN), using an SSBN algorithm [48]. A given MTheory can be used to construct many different SSBNs for different situations. To understand how this works, consider Figure 1, which shows an MTheory called the Danger Assessment MTheory. This MTheory contains seven MFrags: Speed, ImageTypeReport, VehicleObject, Danger, Weather, Region, and Reference. An MFrag may contain three types of random variables: context RVs, denoted by green pentagons, resident RVs, denoted by yellow ovals, and input RVs, denoted by gray trapezoids. Each MFrag defines local probability distributions for its input RVs. These distributions may depend on the input RVs, whose distributions are defined in other MFrags. Context RVs express conditions that must be satisfied for the distributions defined in the MFrag to apply. For example, consider the VehicleObject MFrag in the MTheory of Figure 1. This MFrag expresses knowledge of how the vehicle class is related to the terrain type. The context RVs, IsA(obj, VEHICLE), IsA(rgn, REGION), and $r g n=$ Location $(o b j)$, indicate that the ordinary variable obj must refer to a vehicle, the ordinary variable $r g n$ must refer to a region, and the object denoted by obj must be located in the region denoted by rgn. The resident RV VehicleClass(obj) refers to the type of $o b j$. This type is uncertain, with its distribution defined in the MFrag. The distribution depends on the type of terrain in the region, with wheeled vehicles more likely on roads and tracked vehicles more likely on rough terrain. The terrain type is represented by the input RV TerrainType(rgn), whose distribution is defined in the MFrag Region. The distribution for a resident random variable, defined in its home MFrag, is called a class local distribution. For example, the class local distribution (CLD) of VehicleClass(obj), which depends on the type of terrain where the vehicle is located, can be expressed as Listing 1.

This CLD specifies that if the terrain type is Road, then there is an $80 \%$ chance the vehicle is wheeled; and if the terrain type is OffRoad, then there is an $80 \%$ chance the vehicle is tracked. The final clause specifies a distribution if none of these conditions is met, which is equal probabilities for tracked and wheeled vehicles. 
Listing 1. The class local distribution of the VehicleClass(obj) RV.
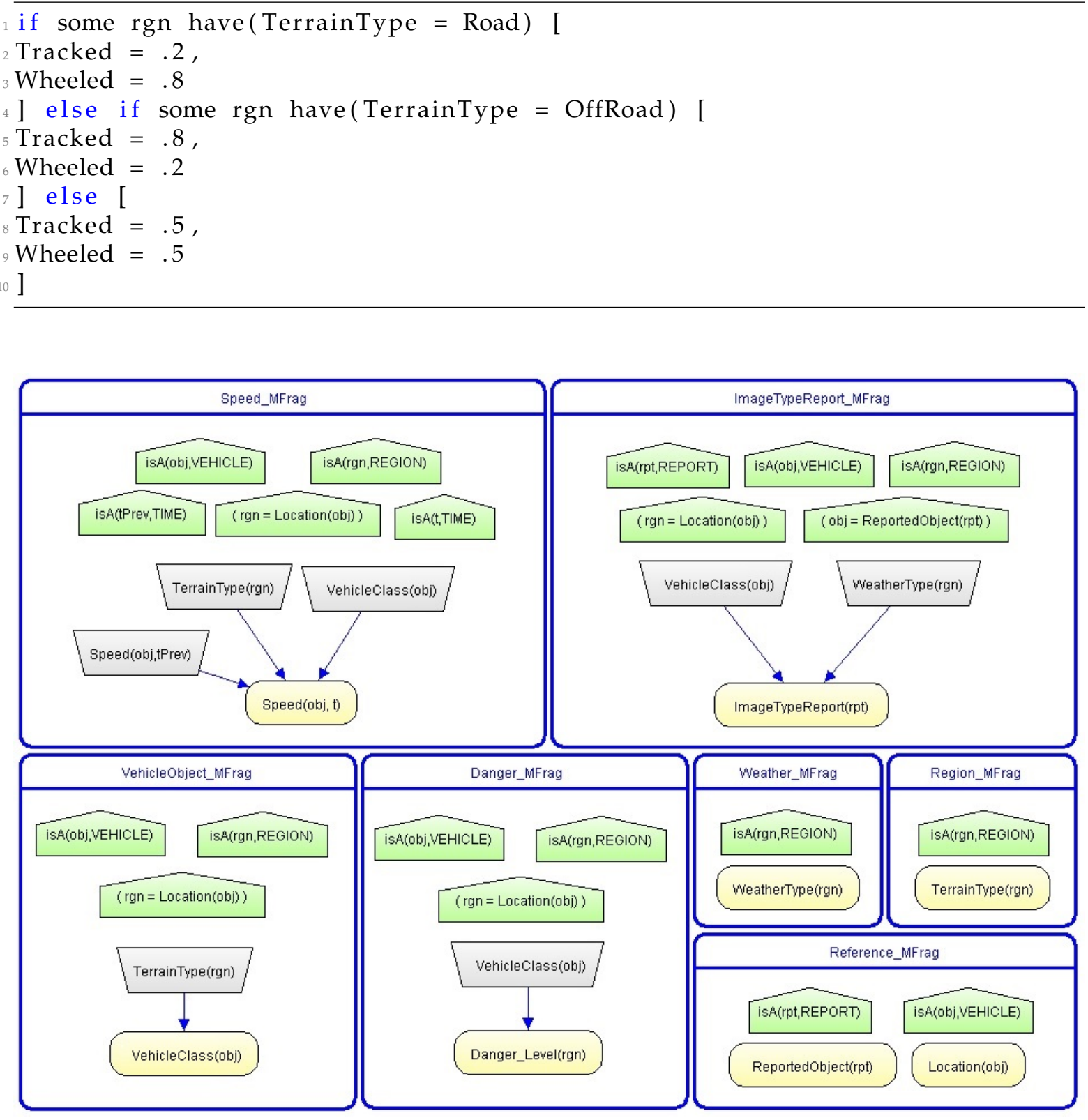

Figure 1. Danger Assessment MTheory.

Formally, an MFrag is defined as follows [48].

Definition 1 (MFrag). An MFrag F, or MEBN fragment, consists of: (i) a set $C$ of context nodes, which represent conditions under which the distribution defined in the MFrag is valid; (ii) a set I of input nodes, which have their distributions defined elsewhere and condition the distributions defined in the MFrag; (iii) a set $\boldsymbol{R}$ of resident nodes, whose distributions are defined in the MFrag; (iv) an acyclic directed graph $G$, whose nodes are associated with resident and input nodes; and (v) a set $\boldsymbol{L}^{\mathrm{C}}$ of CLDs, in which an element of $\boldsymbol{L}^{\mathrm{C}}$ is associated with each resident node.

The nodes in an MFrag are different from the nodes in a common Bayesian network. A node in a common BN represents a single random variable, whereas a node in an MFrag represents a collection of RVs: those formed by replacing the ordinary variables with identifiers of entity instances that 
satisfy the context conditions. To emphasize the distinction, we call the resident nodes MEBN nodes, or MNodes.

MNodes correspond to predicates (for true/false RVs) or terms (for other RVs) of FOL. An MNode is written as a predicate or a term followed by a parenthesized list of ordinary variables as arguments.

Definition 2 (MNode). An MNode N, or MEBN Node, is a random variable N(ff) corresponding to an n-ary function or predicate of $F O L$, a list of $n$ arguments consisting of ordinary variables, a set of mutually exclusive and collectively exhaustive possible values, and an associated CLD. The special values true and false are the possible values for predicates, but may not be possible values for functions.

The RVs associated with the MNode are constructed by substituting domain entities for the $n$ arguments of the function or predicate. The CLD specifies how to define local distributions for these RVs. For example, the node VehicleClass(obj) in Figure 1 is an MNode corresponding to the FOL function VehicleClass (obj). It has two possible values (i.e., Wheeled and Tracked). This MNode is associated with the class local distribution $L^{C}$ in Listing 1 . The MNode is used as a template for the distributions of instance RVs created when an SSBN is constructed from the MFrag associated with the MNode. These instances are formed by substituting identifiers of vehicle objects for the ordinary variable $o b j$.

Definition 3 (MTheory). An MTheory M, or MEBN Theory, is a collection of MFrags that satisfies conditions given in [48] ensuring the existence of a unique joint distribution over its RVs.

An MTheory is a collection of MFrags that defines a consistent joint distribution over RVs describing a domain. The MFrags forming an MTheory should be mutually consistent. To ensure consistency, conditions must be satisfied, such as no-cycle, bounded causal depth, unique home MFrags, and recursive specification condition [48]. No-cycle means that the generated SSBN will contain no directed cycles. Bounded causal depth means that depth from a root node to a leaf node of an instance SSBN should be finite. Unique home MFrags means that each random variable has its distribution defined in a single MFrag, called its home MFrag. Recursive specification means that MEBN provides a means for defining the distribution for an RV depending on an ordered ordinary variable from previous instances of the RV.

The Is $A$ random variable is a special RV representing the type of an entity. IsA is commonly used as a context node to specify the type of entity that can be substituted for an ordinary variable in an MNode.

Definition 4 (IsA Random Variable). An Is $A$ random variable, Is $A(o v, t p)$, is an $R V$ corresponding to a 2-argument FOL predicate. The Is $A R V$ has value true when its second argument tp is filled by the type of its first argument ov and false otherwise.

For example, in the MFrag Danger in Figure 1, IsA(obj, VEHICLE) is an IsA RV. Its first argument $o b j$ is filled by an entity instance and its second argument is the type symbol Vehicle. It has value true when its first argument is filled by an object of the type Vehicle.

\subsection{A Script for MEBN}

Figure 1 shows a graphical representation for an MTheory. In this subsection, we introduce a script representing an MTheory. This script is useful to manage contents of an MTheory. The MTheory Danger Assessment in Figure 1 can be represented by the following script (Listing 2). 
Listing 2. Part of Script MTheory for Danger Assessment.

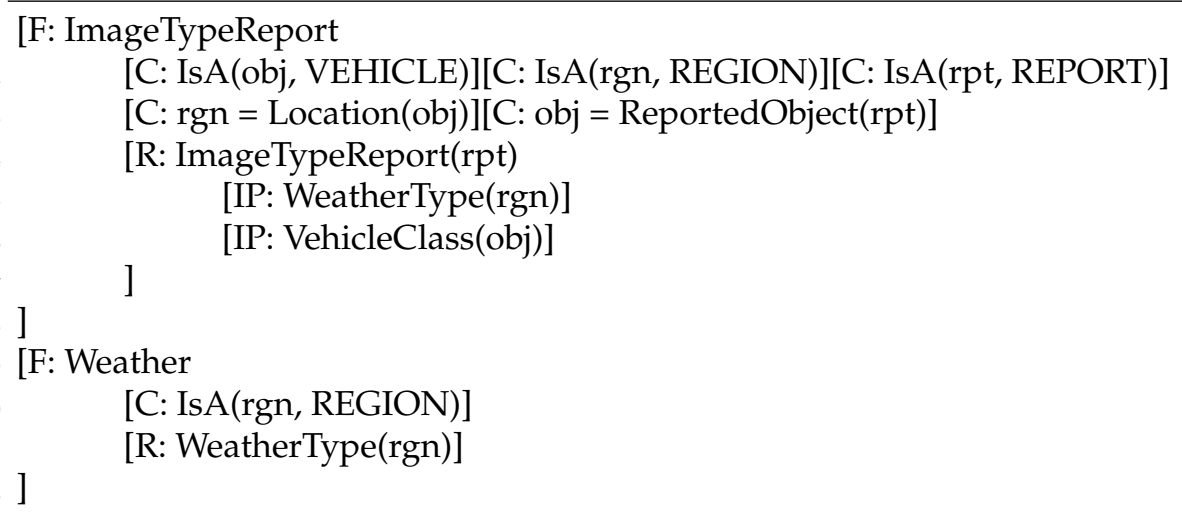

The script contains several predefined single letters (F, C, R, IP, RP, and L). The single letters, F, $\mathrm{C}$, and $\mathrm{R}$ denote an MFrag, a context node, and a resident node, respectively. For a resident node (e.g., $\mathrm{Y}$ ) in an MFrag, a resident parent (RP) node (e.g., $\mathrm{X}$ ), which is defined in the MFrag, is denoted as RP (e.g., [R: Y [RP: X]]). For an input node, we use a single letter IP. Each node can contain a CLD denoted as L. For example, suppose that there is a CLD type called WeatherCLD. If the resident node WeatherType in Line 11 uses the CLD type WeatherCLD, the resident node WeatherType can be represented as [R: WeatherType (rgn) [L: WeatherCLD]].

\subsection{Relational Model}

In 1969, Edgar F. Codd proposed the Relational Model (RM) as a database model based on first-order predicate logic [41-43]. The RM is the most popular database model. A relational database (RDB) is a database that uses the RM to describe and organize data. In the RM, data are organized as a collection of relations. A relation is an abstract definition of a class of entities or a relationship that can hold between classes of entities. An instance of a relation is depicted as a table in which each column is an attribute of the relation and each row, also called a tuple, contains the value of each attribute for an individual entity of the class represented by the relation. An entry in the table, called a cell, is the value of the attribute associated with the column for the entity associated with the row. A key for a relation is one or more attributes that uniquely identify a particular domain entity or row. A primary key uniquely identifies the individual entities in the relation. A foreign key points to the primary key in another relation. The cardinality of a relation is the number of rows in the table, i.e., the number of unique entities of the type represented by the relation. The degree of the relation is the number of columns in the table, i.e., the number of attributes of entities of the type represented by the relation.

Figure 2 shows an illustrative example of an RDB. In the example RDB, there are three relations: Vehicle, Region, and VehicleLocation. We could imagine different situations, each with different vehicles, regions, etc. Each particular situation, such as the one depicted in Figure 2, corresponds to an instance of this relational model. The instance is represented as a table for each of the relations as shown Figure 2, where the columns represent attributes of the relation and the rows represent the attribute values for specific entities. For example, the relation Vehicle has two attributes: VehicleID, which uniquely identifies each individual vehicle, and VehicleClass, which indicates whether the vehicle is tracked or wheeled. The relation VehicleLocation has three attributes: LocatingVehicleID, LocatingTimeID, and Location. The attribute LocatingVehicleID in the relation VehicleLocation is a foreign key pointing to the primary key of the relation Vehicle. A row of the relation VehicleLocation represents a vehicle being located in a region at a point in time. Attributes that are part of the primary key of the relation (e.g., LocatingVehicleID and LocatingTimeID in the relation Location) are denoted by bold, italicized, and underlined letters, while foreign keys which are not part of the primary key of the relation in which 
the foreign keys are used (e.g., Location in the relation VehicleLocation) are denoted by underlined letters. A relation without instances or data-that is, an empty table - is called the relation schema. Hereinafter, for MEBN-RM, we define the elements of RM formally.

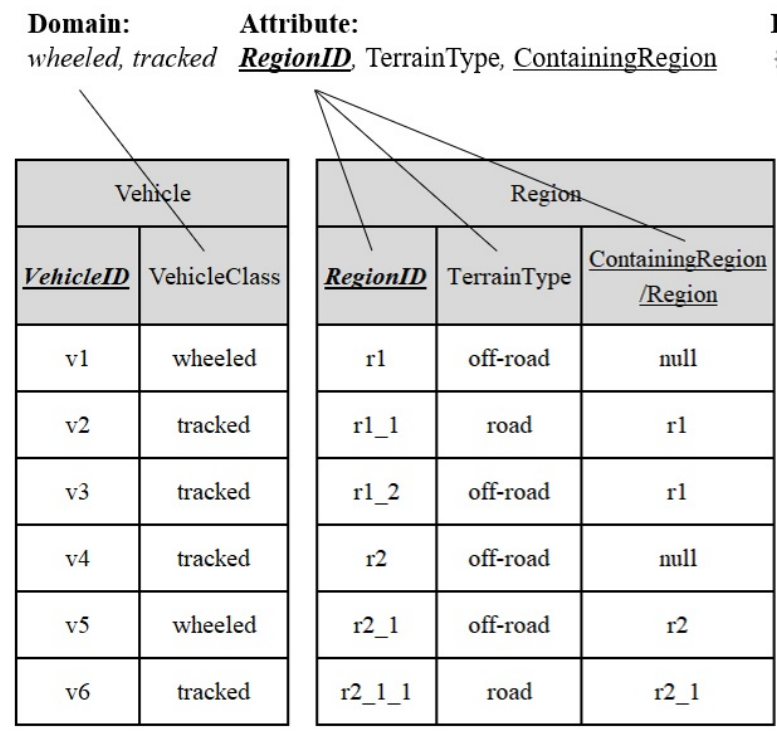

Relation: Vehicle, Region, \& VehicleLocation

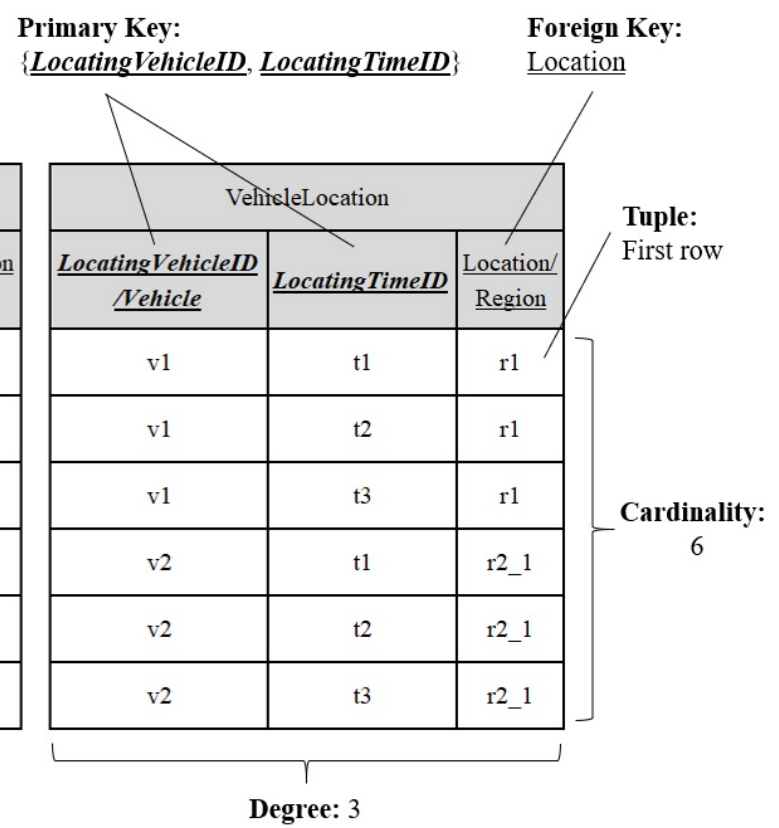

Figure 2. Example of a Part of the Vehicle Identification RDB.

Definition 5 (Relation Schema). A relation schema, $R S\left[A_{1}: D_{1}, A_{2}: D_{2}, \ldots, A_{n}: D_{n}\right]$, is a set of pairs $A_{i}: D_{i}$, where $A_{1} \neq \ldots \neq A_{n}$ are attribute names, and $D_{i}$ is a set called the domain for an attribute $i$.

For example, the relation schema of Vehicle in Figure 2 is [VehicleID: $\{\mathrm{v} 1, \mathrm{v} 2, \ldots\}$, VehicleClass:\{wheeled, tracked\}], where VehicleID and VehicleClass are attributes with domains \{v1, $\mathrm{v} 2, \ldots\}$ and wheeled, tracked, respectively. Please note that we denote the domain of the attribute by inserting the colon, ":", between the name of the attribute and the name of the domain. This ancillary information can be omitted for brevity (e.g., [VehicleID, VehicleClass]). As another example, the relation schema of the relation VehicleLocation is VehicleLocation[LocatingVehicleID: $\{\mathrm{v} 1, \mathrm{v} 2, \ldots\}$, LocatingTimeID: $\{\mathrm{t} 1, \mathrm{t} 2, \ldots\}$, Location: $\left.\left\{\mathrm{r} 1, \mathrm{r} 1 \_1, \mathrm{r} 1 \_2, \ldots\right\}\right]$, where LocatingVehicleID, LocatingTimeID, and Location are attributes, with domains $\{\mathrm{v} 1, \mathrm{v} 2, \ldots\},\{\mathrm{t} 1, \mathrm{t} 2, \ldots\}$, and $\left\{\mathrm{r} 1, \mathrm{r} 1 \_1, \mathrm{r} 1 \_2, \ldots\right\}$, respectively.

Definition 6 (Key). A key of a relation schema is a set of one or more attributes that uniquely identify a row of the relation.

Definition 7 (Foreign Key). A foreign key, $F K$, of a relation schema, $R S\left[A_{1}, A_{2}, \ldots, A_{n}\right]$, is a subset of the attributes $A_{1}, A_{2}, \ldots, A_{n}$ that uniquely identifies a row of another relation.

The relation VehicleLocation of Figure 2 has two foreign keys (i.e., LocatingVehicleID/Vehicle and Location/Region). Here, we use the "/" symbol followed by the relation name to indicate the relation to which the foreign key points, i.e., the foreign key LocatingVehicleID refers to the relation Vehicle. A relation schema containing a foreign key is called a target relation schema for the foreign key, while a relation schema which is referenced by the foreign key is called a home relation schema. For example, the relation schema VehicleLocation is the target relation schema for the attribute LocatingVehicleID, while the relation schema Vehicle is the home relation schema of the attribute. If the target and home relation schema are same, the foreign key and primary key are the same. In this case, the foreign key is called a recursive foreign key. 
Definition 8 (Primary Key). A primary key, $P K$, of a relation schema $R S\left[A_{1}, A_{2}, \ldots, A_{n}\right]$ is a selected subset of the attributes $A_{1}, A_{2}, \ldots, A_{n}$ that uniquely identifies each tuple in the $R S$.

The relation VehicleLocation of Figure 2 has a PK composed of two attributes (i.e., LocatingVehicleID/Vehicle and LocatingTimeID). Each tuple of the relation VehicleLocation is uniquely identified by these two arguments. Please note that the attribute Location/Region in the relation is not used as the PK in the target relation, but it uniquely identifies each tuple in its home relation (i.e., the relation Region); therefore, it is a foreign key. This kind of key is called a Non-Primary Foreign Key.

Definition 9 (Non-Primary Foreign Key). A Non-Primary Foreign Key, NK, is a Foreign Key that is not used for a $P K$ in a target relation.

The attribute ContainingRegion of the relation Region of Figure 2 is another example of a non-primary foreign key since the home relation schema of the attribute ContainingRegion is the relation Region and it is not used for the PK of the target relation.

Definition 10 (Non-Foreign-Key Attribute). A Non-Foreign-Key Attribute, A, is an attribute which is not a foreign key.

For example, in Figure 2, the VehicleClass and TerrainType attribute are non-foreign-key attributes since they are not foreign keys.

Definition 11 (Original Primary Key). An Original Primary Key, OK, is a PK that is not a foreign key in any target relation.

A PK in one relation can be imported from the PK in another relation (i.e., foreign \& primary key), while an original primary key is not originated from other PK in another relation. Examples for the original primary key can include VehicleID in the relation Vehicle, RegionID in the relation Region, and LocatingTimeID in the relation Location.

Definition 12 (Entity Relation Schema). An entity relation schema, ERS, is a relation schema containing an original primary key that is not a foreign key and consists of exactly one attribute.

In Figure 2, the Vehicle and Region relation schema are ERSs. An ERS represents a type of entity. An original primary key in an ERS is a field that holds an identifier that uniquely identifies an instance of the entity type.

Definition 13 (Relationship Relation Schema). A relationship relation schema, $R R S$, is a relation schema containing a PK consisting of attributes which are foreign keys pointing to ERSs.

Therefore, a relationship relation represents a relationship for one entity type (i.e., a unary relation) or a relationship between entities of more than two entity types. In Figure 2, the relation schema VehicleLocation is a relationship relation schema, if we assume that there is a Time ERS and the attribute LocatingTimeID points to the Time ERS.

Definition 14 (Relation Instance). A relation instance, $R I$, of a relation schema, $R S\left[A_{1}, A_{2}, \ldots, A_{n}\right]$, is specified by a table with $n$ columns and $m$ rows, $\left\{\left\{d_{11}, d_{21}, \ldots, d_{n 1}\right\}, \ldots\left\{d_{1 m}, d_{2 m}, \ldots, d_{n m}\right\}\right\}$, where $A_{i}$ is an attribute of $R S, d_{i j} \in \operatorname{Dom}\left(A_{i}\right)$. The relation instance represents a set of $m$ specific entities of the class represented by the relation. 
For example, in the relation Vehicle, there are six rows (i.e., $\{\{\mathrm{v} 1$, wheeled $\},\{\mathrm{v} 2$, tracked $\},\{\mathrm{v} 3$, tracked $\},\{\mathrm{v} 4$, tracked $\},\{\mathrm{v} 5$, wheeled $\},\{\mathrm{v} 6$, tracked $\}\})$. The instance of the relation Vehicle refers to these six rows or tuples.

Definition 15 (Relational Database Schema). A relational database schema, $R D B S\left[R S_{1}, R S_{2}, \ldots, R S_{n}\right]$, is a set of relation schemas.

For example, the table headers of Figure 2 describe the relational database schema, RDBS[Vehicle, Region, VehicleLocation].

Definition 16 (Relational Database). A relational database, $R D B\left[R I_{1}: R S_{1}, R I_{2}: R S_{2}, \ldots, R I_{n}: R S_{n}\right]$, is a set of pairs $R I_{i}: R S_{i}$, where $R S_{i}$ denotes a relation schema and $R I_{i}$ denotes a relation instance of $R S_{i}$.

For example, the tables of Figure 2 describe the relational database RDB $[\{\mathrm{v} 1$, wheeled $\} \ldots\{\mathrm{v} 6$, tracked $\}$ :Vehicle, $\left\{\{\mathrm{r} 1\right.$, offroad, null $\} \ldots\left\{\mathrm{r} 2 \_1 \_1\right.$, road, r2_1\}\}:Region, $\left\{\{\mathrm{v} 1, \mathrm{t} 1, \mathrm{r} 1\} \ldots\left\{\mathrm{v} 2, \mathrm{t} 3, \mathrm{r} 2 \_1\right.\right.$ \}\}:VehicleLocation].

In the relational model, normalization is an operation performed on an RDB to make it more manageable by minimizing redundancy of elements and reducing dependency between attributes [41]. Several normal forms have been suggested such as First $\sim$ Fifth normal form and Boyce-Codd Normal Form (BCNF) [41-43,49-51].

\section{MEBN-RM}

Both MEBN and RM have their theoretical basis in FOL, and both represent entities in a domain and relationships among them. We would like to be able to use data stored in an RDB to learn the joint distribution represented by an MTheory. To do this, we need a mapping from elements of RM to elements of MEBN. MEBN-RM provides such a mapping. MEBN-RM contains four levels of mapping from elements of a relational database to elements of an MTheory. In the first level, an entity mapping between an ERS and an entity in MEBN is defined. In the second level, a resident node mapping is defined. In the third level, a relation and MFrag mapping is defined. In the fourth level, a relational database schema and MTheory mapping is defined. Before discussing these mappings, some ingredients and assumptions are discussed in this section. The following Listing 3 from the Vehicle Identification RDB in Section 2 is used for an illustrative example through Section 3. In the example RDBS, there are four relations: Vehicle, Region, VehicleLocation, and Follow. The relation Vehicle has two attributes: VehicleID and VehicleClass. The relation Region has three attributes: RegionID, TerrainType, and ContainingRegion. The relation VehicleLocation has three

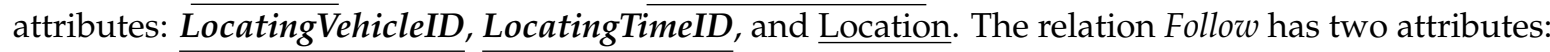
Following VehicleID and LeadingVehicleID.

Listing 3. Vehicle Identification.

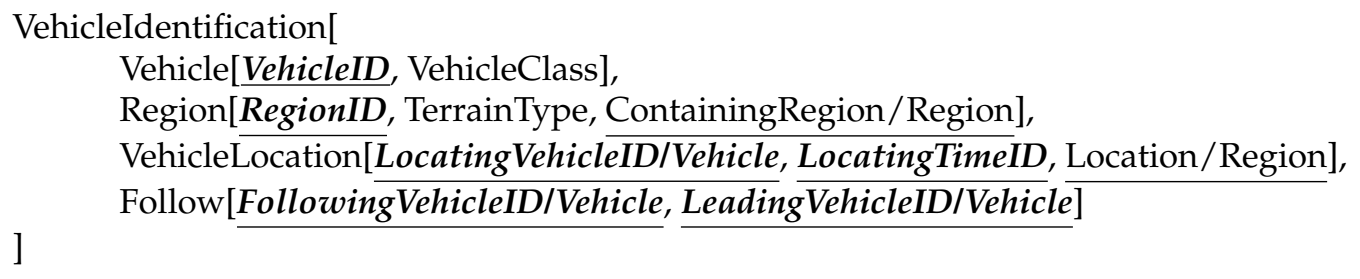

As we saw in Section 2, an attribute in a relation can be a Primary key (PK), Non-Foreign-Key Attribute (NF), or Non-Primary Foreign Key (NK). For example, in the relation Region, the attribute 
RegionID is PK, the attribute TerrainType is NF, and the attribute ContainingRegion is NK. Because each of these types of attribute plays a different role in MEBN-RM, we distinguish them from each other.

In Section 2, Definition 5 defined a relation schema as a set of pairs consisting of an attribute name and a domain (i.e., RS[ $\left.A_{1}: D_{1}, A_{2}: D_{2}, \ldots, A_{n}: D_{n}\right]$, where $A_{i}$ is the $i$-th attribute name and $D_{i}$ is the domain for the attribute $i$ ). The attributes $\left[\mathrm{A}_{1}, \mathrm{~A}_{2}, \ldots, \mathrm{A}_{n}\right]$ can be grouped into three disjoint and exhaustive subsets: PK, NF, and NK, where PK is the set of attributes in a primary key, NF is the set of non-foreign-key attributes, and NK is the set of attributes in a non-primary foreign key.

A variety of relations can be formed in accordance with the following restrictions on the attributes in these subsets. A PK in a relation cannot be empty; however, an NF, NK, or both in a relation may be empty. Therefore, a relation can be one of four types: (1) RS[PK] denotes a relation schema containing only a primary key, (2) RS[PK, NF] denotes a relation schema containing only a primary key and non-foreign-key attributes, (3) RS[PK, NK] denotes a relation schema containing only a primary key and non-primary foreign key attributes, and (4) RS[PK, NF, NK] denotes a relation schema containing a primary key, non-foreign-key attributes, and non-primary foreign key attributes. We define the mapping between RM and MEBN for each of these four types of relation.

We start by assuming that all relations in the RDB are in at least first normal form [52]. Therefore, no relation may contain multiple values in a row (and domain) of an attribute of the relation. To accord with the formalism of MEBN, we introduce a new kind of normalization for MEBN-RM, which we call Entity-Relationship Normalization.

An MTheory developed from an RDB represents entities. We would like to derive these entities from the RDB. We can do this by defining an entity type in MEBN for each entity relation. This entity type can then be referenced in another relation by using the PK of the entity relation as a foreign key in a referring relation. For example, we can identify an entity of type Vehicle corresponding to the relation Vehicle from Figure 2, and use the PK VehicleID to refer to a specific vehicle instance.

For this method to produce a clearly defined mapping, we must make sure that all entity types we wish to represent in the MEBN model are represented as entity relations. As an example of a problem that can occur if this practice is not followed, consider an example of a relationship relation that contains a PK consisting of two attributes that are not foreign keys. For example, we might represent patrol assignments using a PatrolAssignment relation with attributes PatrolDriver, PatrolNavigator, PatrolVehicle/Vehicle, and PatrolRegion/Region. The latter two attributes, the vehicle used and the region patrolled, are foreign keys pointing to the Vehicle and Region relations, respectively. The first two refer to the driver and navigator. These refer to soldiers. If we used this two-attribute primary key to define an entity type, we might erroneously create two different types, when the intention was that both would be filled by an entity of type Soldier. To address this issue, we would create a Soldier relation with its own original primary key (Definition 11), and redefine PatrolDriver and PatrolNavigator as foreign keys pointing to the relation Soldier (i.e., PatrolDriver/Soldier, PatrolNavigator/Soldier, PatrolVehicle/Vehicle, and PatrolRegion/Region).

To formalize this idea, we define Entity-Relationship Normalization to ensure that each entity instance is uniquely identified and to clarify which attributes in a relation correspond to entities in MEBN.

Definition 17 (Entity-Relationship Normalization). A relation is in Entity-Relationship Normal Form if either it is an ERS in Definition 12 or a relationship relation schema in Definition 13.

In the example of Figure 2, the relation VehicleLocation contains a PK consisting of the attributes LocatingVehicleID and LocatingTimeID. LocatingVehicleID is a foreign key, while LocatingTimeID is not. Therefore, in Entity-Relationship Normalization, a new relation for LocatingTimeID should be added (e.g., the relation Time) and the attribute LocatingTimeID should be changed to a foreign key pointing to the new relation Time. As a result, of this transformation, there are three relations (Vehicle, Region, 
and Time) in which a PK for each of them consists of a single attribute. These relations are used to identify entities in an MEBN model. Thus, there are three entities; Vehicle, Region, and Time.

MEBN-RM provides a conversion from a relation schema (RS) in Entity-Relationship Normal Form to a partial MFrag containing a set of context and resident nodes. Full conversion from a relation instance to a complete MFrag (i.e., context nodes, resident nodes, input nodes, a directed acyclic graph, and local distributions) requires augmenting MEBN-RM with either a human modeler or a machine learning algorithm. Hence, in the following sections, MFrag should be taken to mean a partial MFrag.

\subsection{Entity Mapping}

In MEBN, an entity is a unique kind of thing which exists distinctly and independently, and can be instantiated as an object in the world. For example, from a person entity, various person instances can be defined (e.g., John and Mathew). In RM, an entity relation, a relation containing an original primary key consisting of exactly one attribute, represents a kind of thing that exists uniquely and independently. In MEBN-RM, an entity relation or a non-foreign-key attribute can be mapped to an entity in MEBN as defined by the following.

Definition 18 (ERS to Entity Mapping). An ERS to entity mapping is a mapping in which an entity relation schema, ERS, in an RDBS in Entity-Relationship Normal Form is mapped to an entity, E, denoted by ERS $\mapsto E$ ( $A \mapsto B$ means $A$ is mapped to $B$ ).

For example, the ERS (Definition 12) Vehicle can be mapped to an entity VEHICLE. In MEBN-RM, entities are written as strings of uppercase letters.

\subsection{Resident Node Mapping}

In MFrags, a resident node can be described as Function or Predicate of FOL. MEBN allows the modeler to specify a probability distribution for the truth value of a predicate or the value of a function. Formulas are not probabilistic and are defined by built-in MFrags [48]. In this section, we describe the correspondence between functions and predicates in FOL and relations in RM.

Table 1 shows the two types of the resident node with examples from the RDBS Vehicle Identification. These are discussed in the next subsection.

Table 1. Resident Node Types on MEBN-RM.

\begin{tabular}{ccc}
\hline Type & Name & Example \\
\hline 1 & Predicate & Follow(followingvehicleid, leadingvehicleid) \\
2 & Function & VehicleClass(vehicleid), \\
& & $\begin{array}{c}\text { TerrainType(regionid), } \\
\text { ContainingRegion(regionid), } \\
\end{array}$
\end{tabular}

\subsubsection{Predicate}

In FOL, a predicate represents a true/false statement about entities in the domain. It is expressed by a predicate symbol followed by a list of arguments. For example, Follow $(x, y)$ is a predicate that expresses whether a following vehicle indicated by the argument $x$ is following a leading vehicle indicated by the argument $y$. In MEBN, this predicate corresponds to a Boolean RV with possible values true and false. In RM, we can express a predicate as a relation schema in which the attributes are arguments of the predicate, and the rows of the table represent the arguments for which the predicate is true [52]. For example, the relation Follow [FollowingVehicleID, LeadingVehicleID] can be mapped to a predicate, Follow (followingvehicleid, leadingvehicleid). The arguments of this predicate are identical to the set of attributes of the relation to which the predicate refers. A predicate from a relation can 
map to only a true value, because RM does not provide a false value for the predicate. For example, suppose that there is a dataset for the relation Follow $(\{\{v 1, v 2\},\{v 2, v 3\}\})$. This dataset can be a mapped to a set of propositions of the predicate $(\{\operatorname{Follow}(v 1, v 2)=$ true, $\operatorname{Follow}(v 2, v 3)=$ true $\})$.

Table 2 defines the relationship between elements of RM and elements of MEBN for a predicate.

Table 2. Predicate Mapping in MEBN-RM.

\begin{tabular}{cc}
\hline RM & MEBN \\
\hline Name of relation & Name of Predicate \\
Key & Arguments for Predicate \\
Presence of a tuple & true value \\
Absence of a tuple & false value \\
\hline
\end{tabular}

The name of a relation is used for the name of the predicate corresponding to the relation. The attributes of the relation correspond to the arguments of the predicate in sequence. A given tuple can be either present or absent in the RDB. If the tuple is present, a true value for the corresponding predicate can be assigned in the MEBN representation. If the tuple is absent, a false value for the corresponding predicate can be assigned in the MEBN representation. (This convention is used when adopting the closed world assumption, which asserts that all positive cases of a relation are represented in the database, so that absence of an instance implies the corresponding predicate is false. Dropping the closed world assumption could be handled by adding a Truth-Value attribute, with values True and False. With this representation, all cases not appearing in the database would have unknown truth value.) Now, we introduce a predicate resident node mapping.

Definition 19 (Predicate Resident Node Mapping). A predicate resident node mapping is a mapping in which a primary key, $P K$, of a relationship relation schema, $R R S[P K]$, is mapped to a resident node, $R$, denoted by $R R S[P K] \mapsto R\left[R R S\left(A_{1}, A_{2}, \ldots, A_{n}\right)\right]=\{$ true, false $\}$, where $\mathbf{P K}=\left\{K_{1}, K_{2}, \ldots, K_{n}\right\}$ and $K_{i} \mapsto A_{i}$.

For example, the relation schema Follow[FollowingVehicleID, LeadingVehicleID] can be mapped to a predicate resident node denoted by R[Follow (followingvehicleid, leadingvehicleid)].

\subsubsection{Function}

In FOL, a function is a mapping from domain entities called inputs to a value called the output. For example, the function VehicleClass(vehicleid) is a function that maps its argument to wheeled if it is a wheeled vehicle and tracked if it is a tracked vehicle. In RM, a function is represented by a Non-Foreign-Key Attribute (NF) or Non-Primary Foreign Key (NK) of a relation, because both functionally depend on a Primary Key (PK). Thus, a function of a relation maps to its argument(s), the primary key(s) for the relation, to the output, which is the value of the domain of the attribute in the relation.

Table 3 defines the relationship between elements of RM and elements of MEBN for a function. We define a mapping between an element of A or NK of RM, and a function of a resident node of MEBN formally.

Definition 20 (Function Resident Node Mapping). A function resident node mapping is a mapping in which an attribute, $A$, of a relation schema, $R S$, and a primary key, $P K$, of the $R S$ is mapped to a resident node, $R$, of an MFrag, denoted by $R S[\mathbf{P K}, A] \mapsto R\left[A\left(K_{1}, K_{2}, \ldots, K_{n}\right)\right]=\operatorname{Dom}(A)$, where $\mathbf{P K}=\left\{K_{1}, K_{2}, \ldots, K_{n}\right\}$.

For example, the argument of the function VehicleClass(vehicleid) is the PK of the relation Vehicle, and the output is the value (either tracked or wheeled) of the attribute VehicleClass. In other words, Vehicle[VehicleID, VehicleClass] $\mapsto$ R[VehicleClass(vehicleid)] = Dom(VehicleClass). 
Table 3. Function Mapping in MEBN-RM.

\begin{tabular}{cc}
\hline NF or NK of RM & Resident Node of MEBN \\
\hline Non-Foreign-Key Attribute/Non-Primary Foreign Key & Function \\
Primary Key & Arguments of Function \\
Domain of Attribute & Domain of Function \\
\hline
\end{tabular}

\subsection{Relation Schema and MFrag Mapping}

In the previous section, we discussed the mapping between the elements of the relation schema and the elements of the MFrag. In this section, we discuss the mapping between a relation schema and a partial MFrag. It is called RS-MFrag Mapping. For ERS and RRS, we define the RS-MFrag mapping formally.

Definition 21 (RS-MFrag Mapping). An RS-MFrag Mapping is a mapping in which a relation schema, RS, is mapped to a partial MFrag, $F$, denoted by $R S[P K, O] \mapsto F[C, R]$. Here, $C$ denotes a set of context nodes (Definition 1), $\boldsymbol{R}$ denotes a set of resident nodes (Definition 1 ), $P K=\left\{K_{1}, K_{2}, \ldots, K_{n}\right\}$ is the $P K$, and $\boldsymbol{O}=\left\{O_{1}\right.$, $\left.\mathrm{O}_{2}, \ldots, \mathrm{O}_{m}\right\}$, where $\mathrm{O}_{i}$ is the $\mathrm{i}$-th NF or NK attribute. The mapping satisfies the following conditions:

(a) If the RS is an ERS and $|O|>0$, the PK and $\boldsymbol{O}$ of the ERS are mapped to the $\boldsymbol{C}$ and $\boldsymbol{R}$ of the $F$, respectively. This is denoted by ERS[PK, $\boldsymbol{O}] \mapsto F\left[C 1\left[I s A\left(K_{1}, E\left(K_{1}\right)\right)\right], R_{1}\left[O_{1}\left(K_{1}\right)\right], \ldots, R_{m}\left[O_{m}\left(K_{1}\right)\right]\right]$.

(b) If the RS is an RRS and $|\boldsymbol{O}|>0$, the $\boldsymbol{P K}$ and $\boldsymbol{O}$ of the RRS are mapped to the $\boldsymbol{C}$ and $\boldsymbol{R}$ of the $F$, respectively. This is denoted by $R R S[P K, O] \mapsto F\left[C_{1}\left[I s A\left(K_{1}, E\left(K_{1}\right)\right)\right], \ldots, C_{n}\left[I s A\left(K_{n}, E\left(K_{n}\right)\right)\right]\right.$, $\left.R_{1}\left[O_{1}(\mathbf{P K})\right], \ldots, R_{m}\left[O_{m}(\mathbf{P K})\right]\right]$ ( $E(X)$ is the entity type which the attribute $X$ points to).

(c) If the RS is an RRS and $|\boldsymbol{O}|=0$, the $P K$ and RRS are mapped to the $\boldsymbol{C}$ and $\mathbf{R}$ of the $F$, respectively. This is denoted by RRS[PK]$\mapsto F\left[C_{1}\left[I s A\left(K_{1}, E\left(K_{1}\right)\right)\right], \ldots, C_{n}\left[I s A\left(K_{n}, E\left(K_{n}\right)\right)\right], R_{1}[R R S(P K)]\right]$.

Case (a) is that the RS is an ERS and it has at least one attribute which is not used for the PK. In this case, the single attribute $K_{1}$ in $P K$ is used to create the IsA context node, and each attribute in $O$ is mapped to each resident node respectively using the function resident node mapping. For example, the relation Vehicle[VehicleID/Vehicle, VehicleClass] becomes a partial MFrag denoted by F[C[IsA(vehicleid, VEHICLE)], R[VehicleClass(vehicleid)]]. Case (b) is that the RS is an RRS and it has an attribute which is not used for the PK. Each attribute $K_{i}$ in $P K$ is used to create its respective IsA context node and each attribute in $O$ is mapped to a resident node using the function resident node mapping. For example, the relation VehicleLocation[LocatingVehicleID/Vehicle, LocatingTimeID/Time, Location/Region] becomes a partial MFrag denoted by F[C[IsA(locatingvehicleid, VEHICLE), IsA(locatingtimeid, TIME), $\mathrm{R}$ [Location(locatingvehicleid, locatingtimeid)]]. Case (c) is that the RS is the RRS and has no attributes other than the PK. In this case, each attribute $K_{i}$ in $P K$ is used to create the IsA context node and the relation is mapped to a predicate resident node, $\operatorname{RRS}\left(\mathrm{K}_{1}, \mathrm{~K}_{2}, \ldots, \mathrm{K}_{\mathrm{n}}\right)$, using the predicate resident node mapping. For example, the relation Follow [FollowingVehicleID/Vehicle, LeadingVehicleID/Vehicle] becomes a partial MFrag denoted by F[C]IsA(followingvehicleid, VEHICLE), IsA(leadingvehicleid, VEHICLE), R[Follow(followingvehicleid, leadingvehicleid)]].

\subsection{Relational Database Schema and MTheory Mapping}

In the previous section, we discussed the mapping between a relation schema and partial MFrag. In this section, we discuss the mapping between a relational database schema (RDBS) and MTheory (M). It is called RDBS-MTheory Mapping. The mapping produces one MTheory from one relational database schema, denoted by the following.

Definition 22 (RDBS-MTheory Mapping). An RDBS-MTheory Mapping is a mapping in which a relational database schema RDBS is mapped to an MTheory M (Definition 3), denoted by $R D B S\left[R S_{1}, R S_{2}\right.$, 
$\left.\ldots, R S_{n}\right] \mapsto M\left[F_{1}, F_{2}, \ldots, F_{n}\right]$, where $R S_{i}$ is a relation schema in the $R D B S, F_{i}$ is a partial MFrag in the $M$, and $n$ is the number of the relation schemas in the RDBS and the number of the partial MFrags in the $M$, if the RS-MFrag Mapping between $R S_{i}$ and $F_{i}$ is able to be used.

For example, the Vehicle Identification RDBS can be directly an MTheory using the RDBS-MTheory mapping. The relations Vehicle, Region, and VehicleLocation in the RDBS are converted to partial MFrags Vehicle, Region, and VehicleLocation. The following subsection presents a mapping algorithm using MEBN-RM, which is a process to develop an MTheory from data in RM and contains specific sub-steps.

\subsection{MEBN-RM Mapping Algorithm}

In the previous subsections, we discussed the mapping definitions for entities, resident nodes, MFrags, and MTheories. This subsection presents a MEBN-RM mapping algorithm (Algorithm 1) which performs the RDBS-MTheory Mapping in Definition 22 and specifies how to convert an MTheory from a relational database schema using the MEBN-RM definitions. For the MEBN-RM mapping algorithm, we assume that (1) the relational database schema are normalized by Entity-Relationship Normalization (Definition 17), and (2) the list of relation schemas in the relational database schema are sorted by the entity relation schemas (ERS) first and the relationship relation schemas (RRS) second. For the algorithm, let M be an MTheory, M.E be a set of entity types of M, M.F be a set of MFrags, F.C be a set of context nodes in an MFrag F, F.R be a set of resident nodes of F, RDBS be a relational database schema, rs be a relation schema in RDBS, and rs.O be a set of attributes for NF and/or NK of rs. The algorithm takes the relational database schema RDBS as an input and produces the MTheory M as an output.

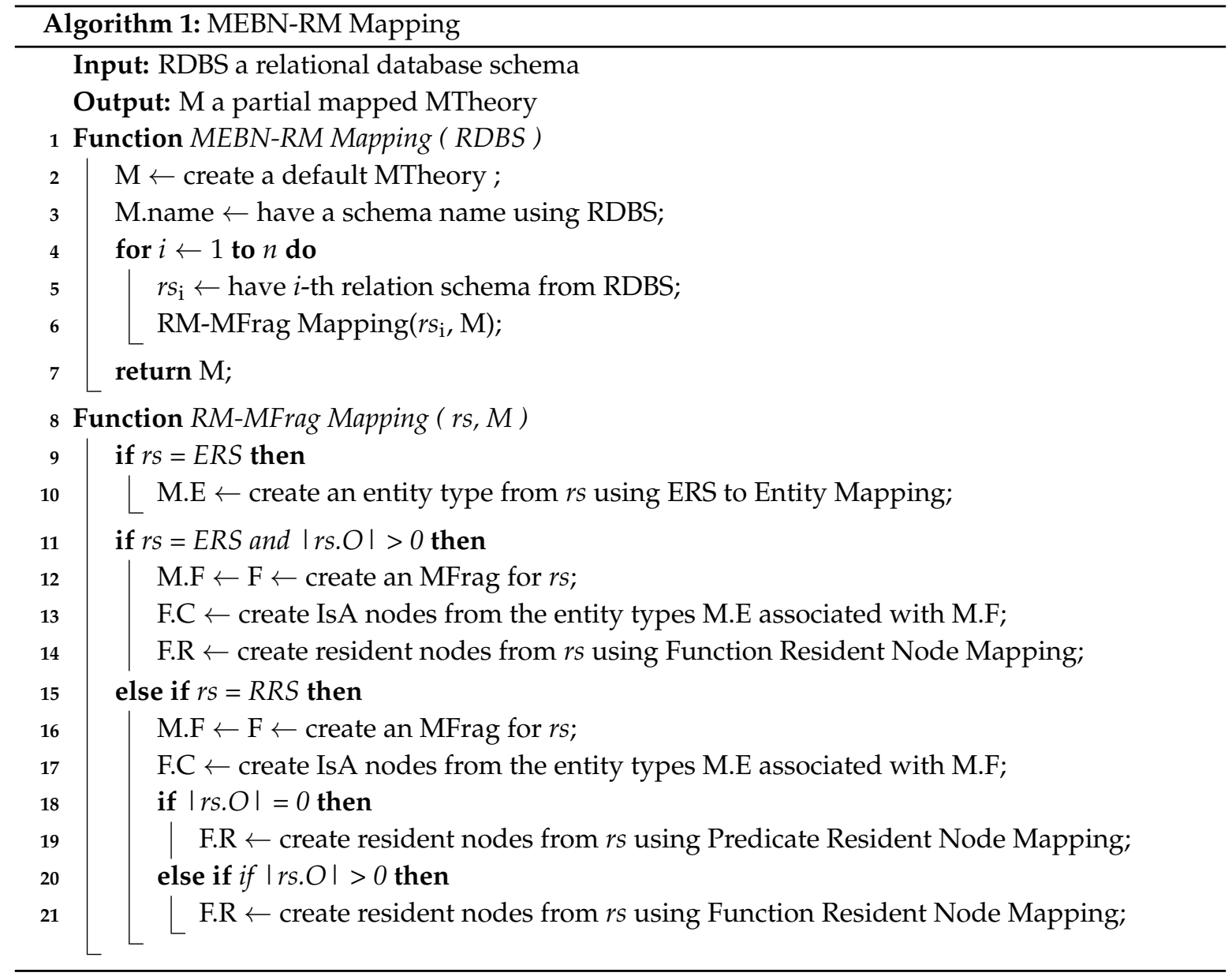


Inputs of this algorithm are a relational database schema RDBS. (2) The algorithm starts with creating a default MTheory M. (3) The name of RDBS is used to create the name of M. (4), (5) All relation schema are investigated from a first relation schema $r s_{1}$ to a last relation schema $r s_{n}$, where $n$ denotes the number of the relation schemas in RDBS. (6) For an $i$-th relation schema, the algorithm performs the procedure RM-MFrag Mapping defined in Definition 21. (9) If the $i$-th relation schema is ERS, (10) the ERS to Entity Mapping (Definition 18) is performed. (11) If the $i$-th relation schema $r s$ is ERS and there is an attribute O for NF or NK, then (12) an MFrag F for the $r s$ is created and added to the set of MFrags of M, (13) IsA context nodes are created from the entity types M.E associated with $\mathrm{F}$ and added to the set of context nodes of $\mathrm{F}$, and (14) resident nodes are created from $r s$ using the function resident node mapping (Definition 20) and added into the set of resident nodes of F. (15) If the $i$-th relation schema $r s$ the is RRS, then performs (16) to (21). (16) An MFrag F for the $r s$ is created and added to the set of MFrags of M. (17) IsA context nodes are created from the entity types M.E associated with $\mathrm{F}$ and added to the set of context nodes of F. (18) If there is no attribute O for NF or NK, (19) the predicate resident node mapping (Definition 19) for $r s$ is performed. (20) If there is an attribute $\mathrm{O}$ for NF or NK, (21) the function resident node mapping (Definition 20) for $r s$ is performed. (7) The algorithm results in the MTheory $\mathrm{M}$.

We consider the complexity of this algorithm in terms of the Big $\mathrm{O}$. The for-loop in Line 3 is the most computationally intensive operation of this algorithm and it is influenced by the number of relations $n$. The complexity of the procedure RM-MFrag Mapping is $\mathrm{O}(k)$, where $k$ is the number of PKs in a relation. Therefore, this algorithm has complexity $\mathrm{O}(n k)$. However, in the most cases, $k$ is not a big number (i.e., $k<n$ ), so we can consider this algorithm as $\mathrm{O}(n)$. We will see experiment results for this in Section 4.2. Listing 4 shows a result for the Listing 3 using the MEBN-RM mapping algorithm.

Listing 4. Vehicle Identification.

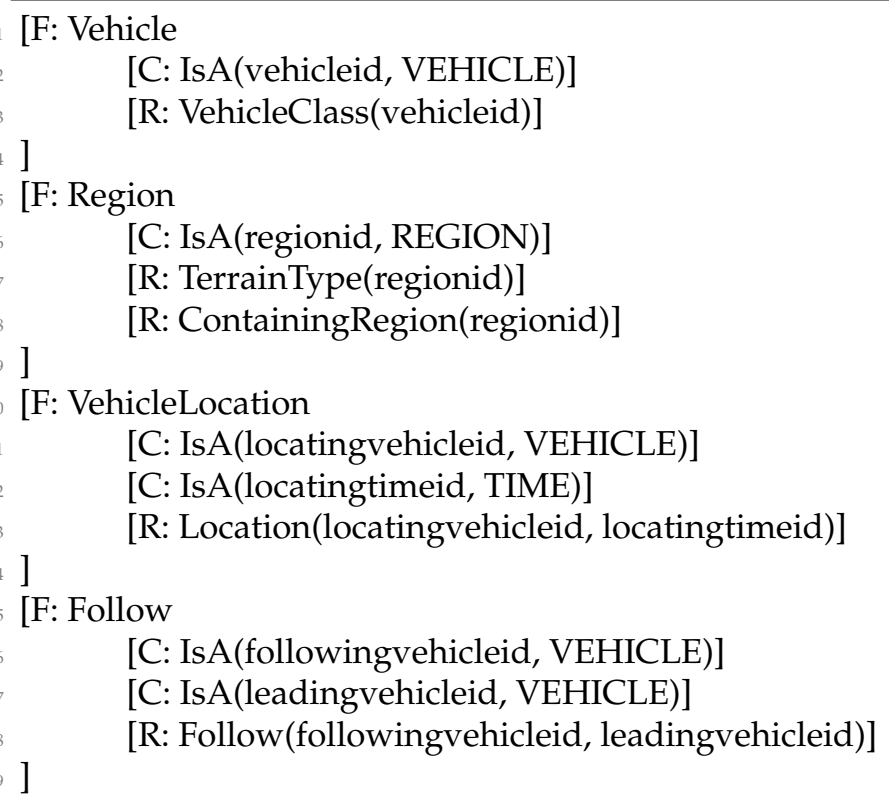

For example, the relation Region in Listing 3 is an ERS, which is mapped to the entity REGION and used to create the IsA context node in Line 6. The relation Region contains an attribute TerrainType which is converted to a resident node TerrainType as a Function in Line 7. Also, the relation Region contains a Non-Primary Foreign Key ContainingRegion which is mapped to a resident node ContainingRegion as a Function in Line 8. 


\section{Experiment for MEBN-RM}

In this section, we present an experiment to evaluate the performance of the MEBN-RM algorithm in terms of mapping speed and quality. The MEBN-RM algorithm was implemented on an open-source program. First this program is introduced and then the experiment is presented.

\subsection{MEBN-RM Tool}

MEBN-RM Tool is a JAVA-based open-source program that can be used to create an MTheory script from a relational schema. MEBN-RM Tool can be commonly used in MEBN learning or MEBN modeling. MEBN-RM Tool is implemented in the MEBN-RM mapping algorithm in Section 3.5. This enables rapid development of an MTheory script by clicking a button in the tool. The current version of MEBN-RM Tool uses MySQL, an open-source relational database management system, to take the relational schema. The most recent version and source code of MEBN-RM Tool are available online at the GMU-HMLP GitHub repository (https://github.com/pcyoung75/GMU_HMLP.git). Once we obtain MEBN-RM Tool we are ready to select a relational database and convert it to an MTheory script. MEBN-RM Tool contains two panels: (1) a left tree panel shows a list of relational databases and (2) a right panel shows a result MTheory script. By selecting a database and clicking the select button in the tool, the MEBN-RM mapping performs and produces a result MTheory script.

\subsection{Experiment}

We conducted the experiment to evaluate the performance of the MEBN-RM algorithm in terms of the mapping time and accuracy. The mapping time is the time it takes to map from a relational database to an MTheory script. The mapping accuracy means how correctly the MTheory script was mapped from the relational database. For this, we compared both elements from the MTheory script and the relational database. For the test relational databases, Relational Learning Repository [53], which contains more than 70 relational databases from the real world or the simulation, was used. For the experiment, 10 real-world relational databases (see Table 4) from 8 domains (Education, Financial, Entertainment, Government, Industry, Kinship, Medicine, and Social) were chosen. These relational databases satisfied Entity-Relationship Normalization (Definition 17), so the normalization step was not required. The experiment was run on a $3.40 \mathrm{GHz}$ Intel Core i7-3770 processor.

Table 4. 10 Real-World Relational Databases with Experiment Results for the Mapping Time.

\begin{tabular}{|c|c|c|c|c|c|c|c|c|}
\hline$\#$ & Name & Domain & $\begin{array}{c}\text { \# of RS } \\
\text { (Definition 5) }\end{array}$ & $\begin{array}{c}\text { \# of ERS } \\
\text { (Definition 12) }\end{array}$ & $\begin{array}{c}\text { \# of RRS } \\
\text { (Definition 13) }\end{array}$ & $\begin{array}{c}\text { \# of } \\
\text { Attributes }\end{array}$ & $\begin{array}{c}\text { \# of } \\
\text { Primary Keys } \\
\text { (Definition 8) }\end{array}$ & $\begin{array}{c}\text { Mapping } \\
\text { Time (Second) }\end{array}$ \\
\hline 1 & Stats & Education & 8 & 8 & 0 & 71 & 8 & 0.0597 \\
\hline 2 & Financial & Financial & 8 & 8 & 0 & 55 & 8 & 0.0498 \\
\hline 3 & MovieLens & Entertainment & 7 & 4 & 3 & 24 & 10 & 0.0445 \\
\hline 4 & LegalActs & Government & 5 & 2 & 3 & 33 & 7 & 0.0334 \\
\hline 5 & SAT & Industry & 36 & 3 & 33 & 69 & 37 & 0.1656 \\
\hline 6 & Dunur & Kinship & 17 & 1 & 16 & 34 & 33 & 0.0726 \\
\hline 7 & Elti & Kinship & 11 & 1 & 10 & 22 & 21 & 0.0503 \\
\hline 8 & Bupa & Medicine & 9 & 2 & 7 & 16 & 9 & 0.0383 \\
\hline 9 & Pima & Medicine & 9 & 1 & 8 & 18 & 9 & 0.0417 \\
\hline 10 & Facebook & Social & 2 & 1 & 1 & 265 & 3 & 0.0359 \\
\hline
\end{tabular}

\subsubsection{Mapping Time}

Table 4 shows 10 relational databases with the name, domain, and number of attributes/relations. Each relational database had different attribute and relation features, so the following factors were used to investigate the mapping time: (1) the number of relations and (2) the number of attributes. Table 4 also shows the experiment results for the mapping time over the different number of attributes and relations in each of the 10 real-world relational databases. The correlation coefficient for the mapping time over the number of attributes was -0.033 , while the correlation coefficient for the mapping time over the number of relations was 0.97 (see Figure 3). 


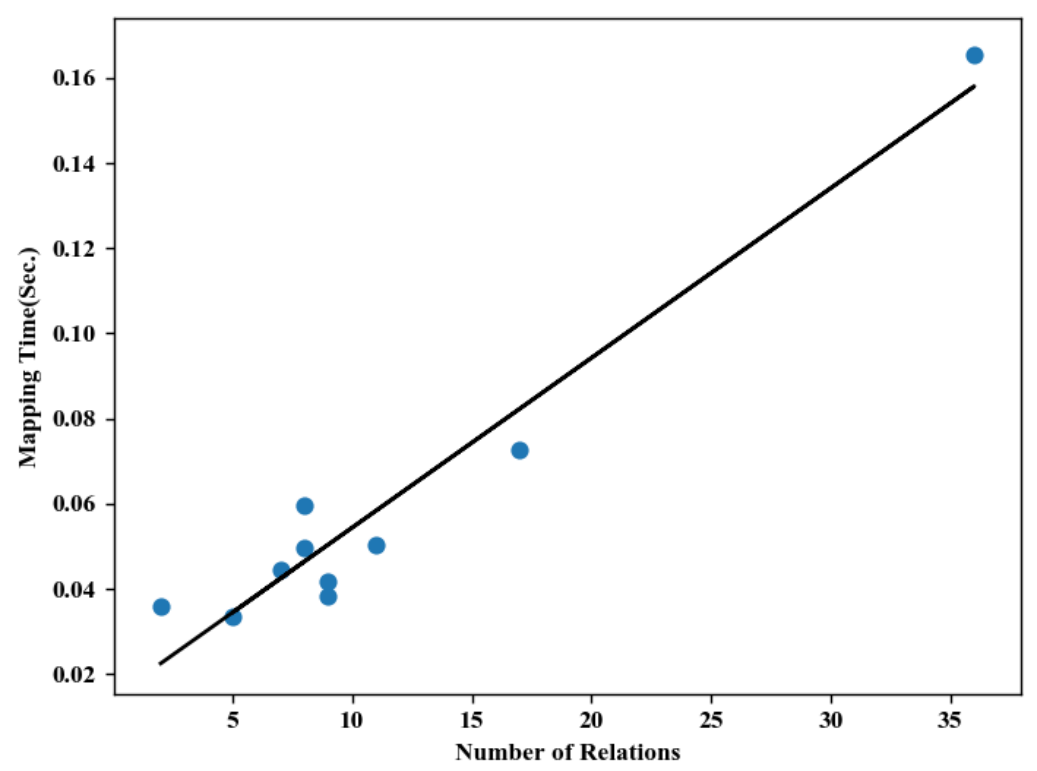

Figure 3. Mapping Time over the Number of Relations.

The increase in mapping time was linear in the number of relations. However, the acceptable speed of the algorithm alone is not sufficient. The accuracy for the mapped results is also essential.

\subsubsection{Mapping Accuracy}

The mapping accuracy for the MEBN-RM algorithm can be evaluated by comparing between the numbers of elements in a source RDB and the numbers of elements in a target MTheory. For example, an ERS (Definition 12) in the RDB and an entity in the MTheory are mapped to one-to-one. An ERS containing NK (Definition 9) or A (Definition 10) is mapped to an MFrag. Table 5 shows the numbers of elements in the target MTheories. These elements were generated as we expected.

Table 5. 10 Real-World Relational Databases with Elements of Its Mapped MTheory.

\begin{tabular}{cccccc}
\hline$\#$ & Name & \# of Entity & $\begin{array}{c}\text { \# of MFrag } \\
\text { (Definition 1) }\end{array}$ & $\begin{array}{c}\text { \# of Resident Node } \\
\text { (Definition 2) }\end{array}$ & $\begin{array}{c}\text { \# of IsA Nodes } \\
\text { (Definition 4) }\end{array}$ \\
\hline 1 & Stats & 8 & 8 & 63 & 8 \\
2 & Financial & 8 & 8 & 47 & 8 \\
3 & MovieLens & 4 & 7 & 14 & 10 \\
4 & LegalActs & 2 & 5 & 28 & 7 \\
5 & SAT & 3 & 33 & 33 & 34 \\
6 & Dunur & 1 & 16 & 16 & 32 \\
7 & Elti & 1 & 10 & 10 & 20 \\
8 & Bupa & 2 & 7 & 7 & 7 \\
9 & Pima & 1 & 9 & 9 & 3 \\
10 & Facebook & 1 & 2 & 263 & \\
\hline
\end{tabular}

We also applied MEBN-RM to two projects. Section 5 introduces the projects with specific relational databases and mapping outcomes.

\section{Use Cases}

In this section, we introduce two example use cases using MEBN-RM: a Critical Infrastructure Defense System and a Smart Manufacturing System. 


\subsection{Critical Infrastructure Defense System}

HERALD is a proof-of-concept system designed to prevent attacks to critical infrastructures (CI) through early detection/identification of threatening targets and short-term prediction of the target's activities and threat level for the region where the critical infrastructures are located [23]. In 2014, Samsung Thales coined the design of a critical infrastructure defense system which is the predecessor of the HERALD system. Requirements from Samsung Thales were to design and develop a next-generation system for critical infrastructure defense by means of integration for previous knowledge with evidence from multiple sensors (e.g., MTI (Moving Target Indicator) system, IMINT (Imagery Intelligence) sensor system, and GEOINT (Geospatial Intelligence) system).

HERALD consisted of an inference module, a control module, and a scenario simulator. The inference module used a HERALD MTheory to infer current and future situations. The HERALD MTheory was designed by domain experts and learned using an MEBN learning process (HMLP) [23]. Also, HERALD contained a relational database that was developed by the domain experts and used for a simulation. The HERALD simulator simulated ground truth information of a situation in which our forces and enemies operated against each other. To develop the HERALD MTheory, the relational database was used to develop a partial HERALD MTheory. Figure 4 shows the schema of the relational database.

The relational database schema contained 18 relations (e.g., MTI_Report, Target, and TargetTemporalProperty). For example, the relation MTI_Report represented knowledge from MTI about reported locations (e.g., LatitudeReport) of and reported moving aspects (e.g., DistanceToCIReport) of a target. The relation Target represented knowledge about a target's information that was not time-varying. The relation TargetTemporalProperty represented knowledge about a target's information that was time-varying.

MEBN-RM was used to convert the relational database schema to the partial HERALD MTheory. Listing 5 shows some MFrags of the partial HERALD MTheory. These three MFrags correspond to the three relations in the relational database schema. Please note that each name of the resident nodes is changed to a form that includes an abbreviation derived from the name of the MFrag (e.g., the prefix MR for resident nodes in the MFrag MTI_Report) to prevent construction for resident nodes with the same name. The domain experts, then, used the partial HERALD MTheory to construct a complete HERALD MTheory by adding CLDs for resident nodes and conditional dependence relationships between the resident nodes.

To evaluate the mapping accuracy, we compared the numbers of elements in the source RDB (Figure 4) and the numbers of elements in the target MTheory (Table 6). These elements in Table 6 were mapped as expected. The total mapping time was $0.0136 \mathrm{~s}$ (running on the $3.40 \mathrm{GHz}$ Intel Core i7-3770 processor).

Table 6. The Results of the MEBN-RM Mapping for the Partial HERALD Relational Database (Figure 4) and Its Mapping Time.

\begin{tabular}{ccccc}
\hline \# of Entity & $\begin{array}{c}\text { \# of MFrag } \\
\text { (Definition 1) }\end{array}$ & $\begin{array}{c}\text { \# of Resident Node } \\
\text { (Definition 2) }\end{array}$ & $\begin{array}{c}\text { \# of IsA Nodes } \\
\text { (Definition 4) }\end{array}$ & MappingTime (Second) \\
\hline 7 & 12 & 31 & 21 & 0.0136 \\
\hline
\end{tabular}

\subsection{Smart Manufacturing System}

An MSAW (Predictive Manufacturing Situation Awareness) system as a prototype system was designed and developed to estimate current situations as well as predict future situations for a steel plate manufacturing [27]. The MSAW system was associated with various equipment for steel plate manufacturing (e.g., a reheating furnace, a roughing mill, and a finishing mill) to produce steel plates of good quality (e.g., few defects and required flatness) by taking steel slabs. The goal of the MSAW system was to support four smart functions: Function 1 (Control value reasoning given outputs), 
Function 2 (Optimal control value finding to maximize/minimize objective values (e.g., outputs)), Function 3 (Prediction for future manufacturing situations), and Function 4 (Sensitivity analysis to find defective factors for faulty outputs). The MSAW system supporting the steel plate manufacturing contained an MSAW MTheory which was used to perform the above four functions.

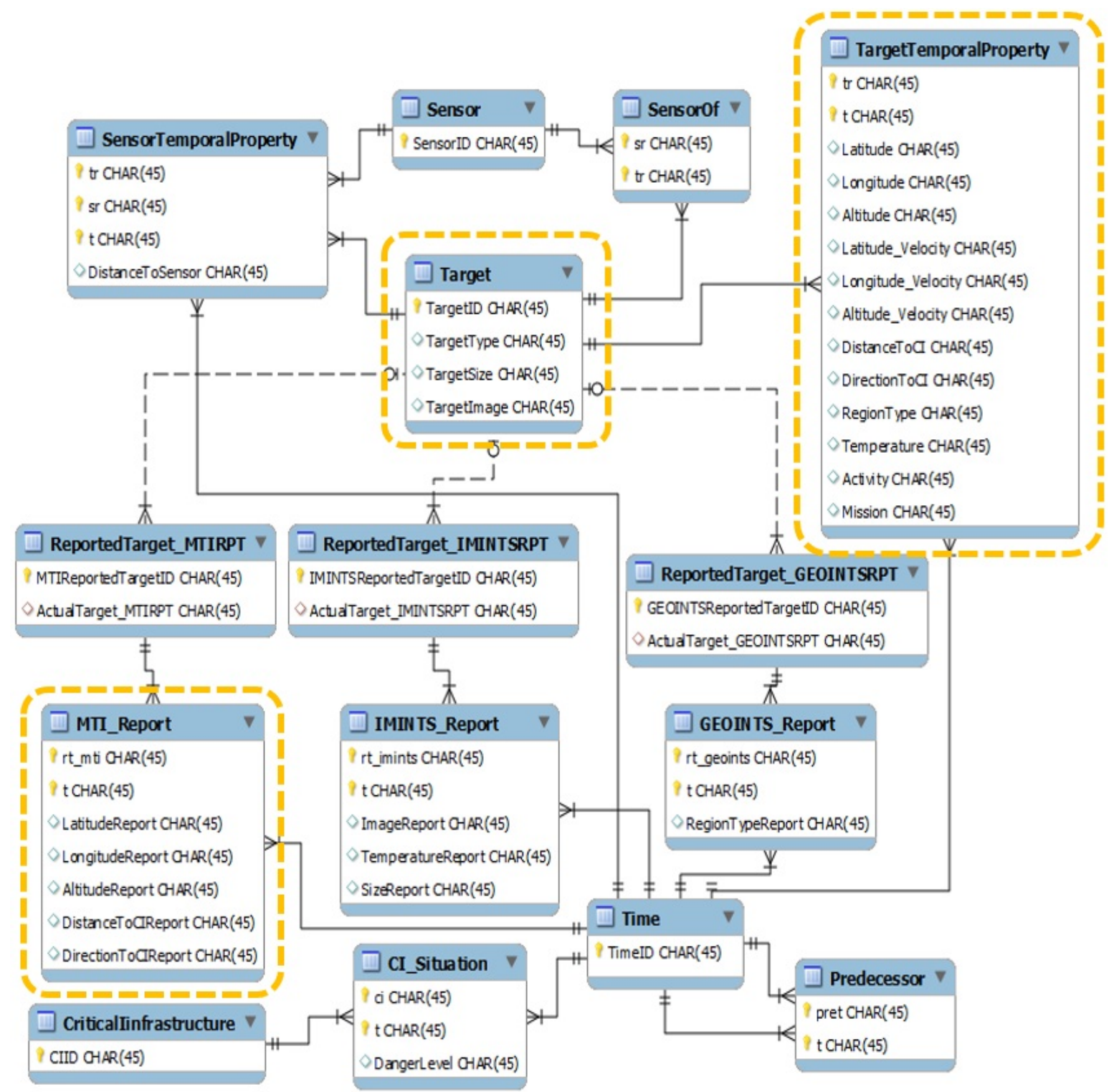

Figure 4. Partial HERALD Relational Database Schema.

The MSAW MTheory was designed by domain experts and learned using data from a simulator. The simulator was designed by domain experts and contained a relational database as shown Figure 5 . The simulator simulated ground truth information for the reheating furnace, the roughing mill, and the finishing mill. The relational database in the simulator was used to develop a partial MSAW MTheory. Figure 5 shows the schema of the relational database.

The relational database schema contained 21 relations. Examples of the relations include heater_item, estimator_item, and heateractuator_item. The relation heater_item represented properties of a slab item heated by the reheating furnace. The relation contained several attributes for the slab item (e.g., attributes SteelGrade, Thickness, and Temperature). The relation estimator_item represented knowledge about an overall situation for manufacturing in terms of total manufacturing cost, total manufacturing time, and total quality rate for products. The relation heateractuator_item represented 
knowledge about control factors and properties for the reheating furnace (e.g., attributes NumberOfSlab and ProductionTime).

MEBN-RM was used to convert the relational database schema to the partial MSAW MTheory. Listing 6 shows some MFrags of the partial MSAW MTheory. These three MFrags correspond to the three relations (i.e., heater_item, estimator_item, and heateractuator_item) in the relational database schema. The domain experts, then, used the partial MSAW MTheory to construct a complete MSAW MTheory by adding local probability distributions for resident nodes and conditional dependence relationships between the resident nodes.

Listing 5. Part of Script MTheory for HERALD corresponding to the Relations shown in Rounded-Dashed Boxes of Figure 4.

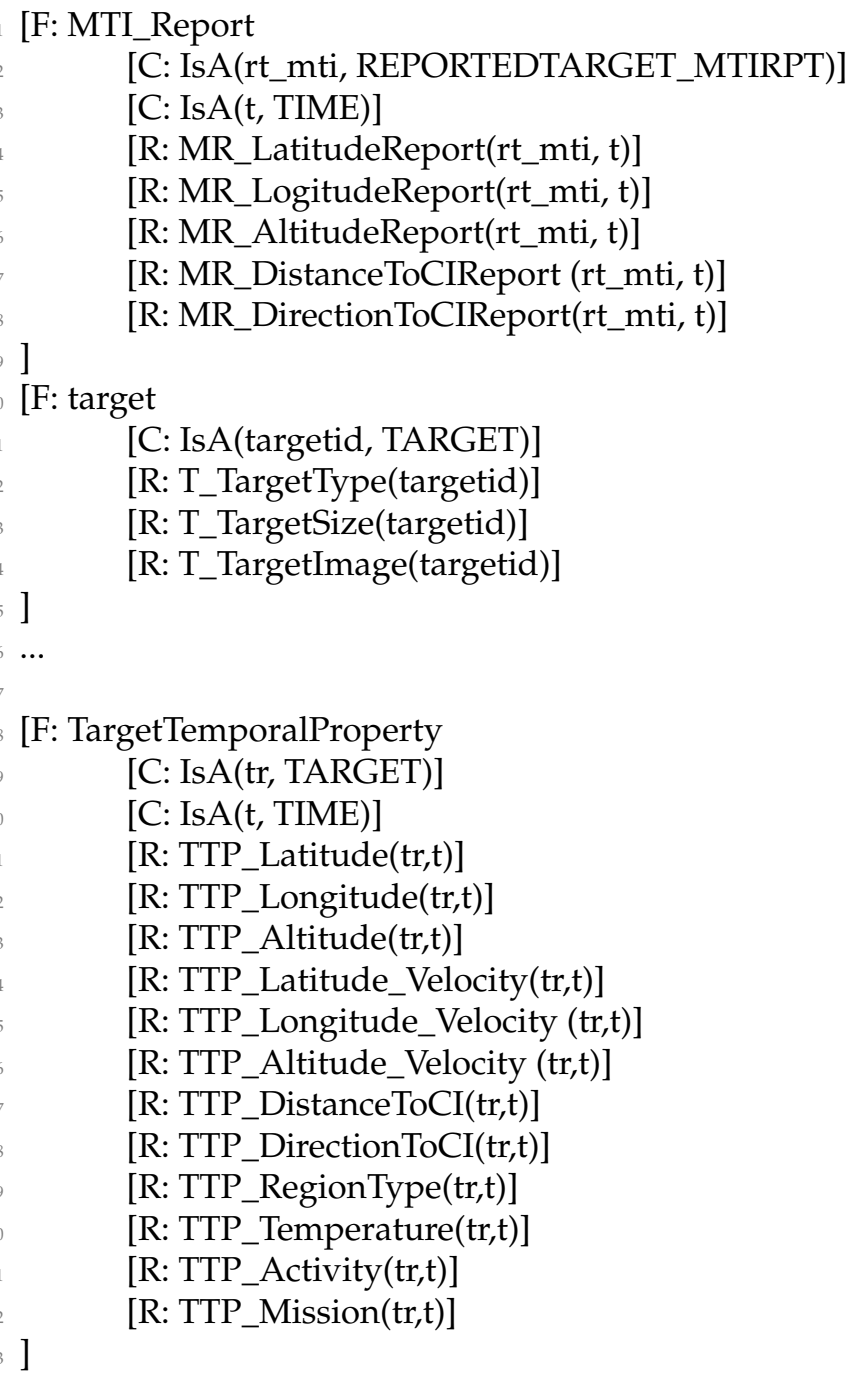


Listing 6. Part of Script MTheory for MSAW corresponding to the Relations shown in Rounded-Dashed Boxes of Figure 5.

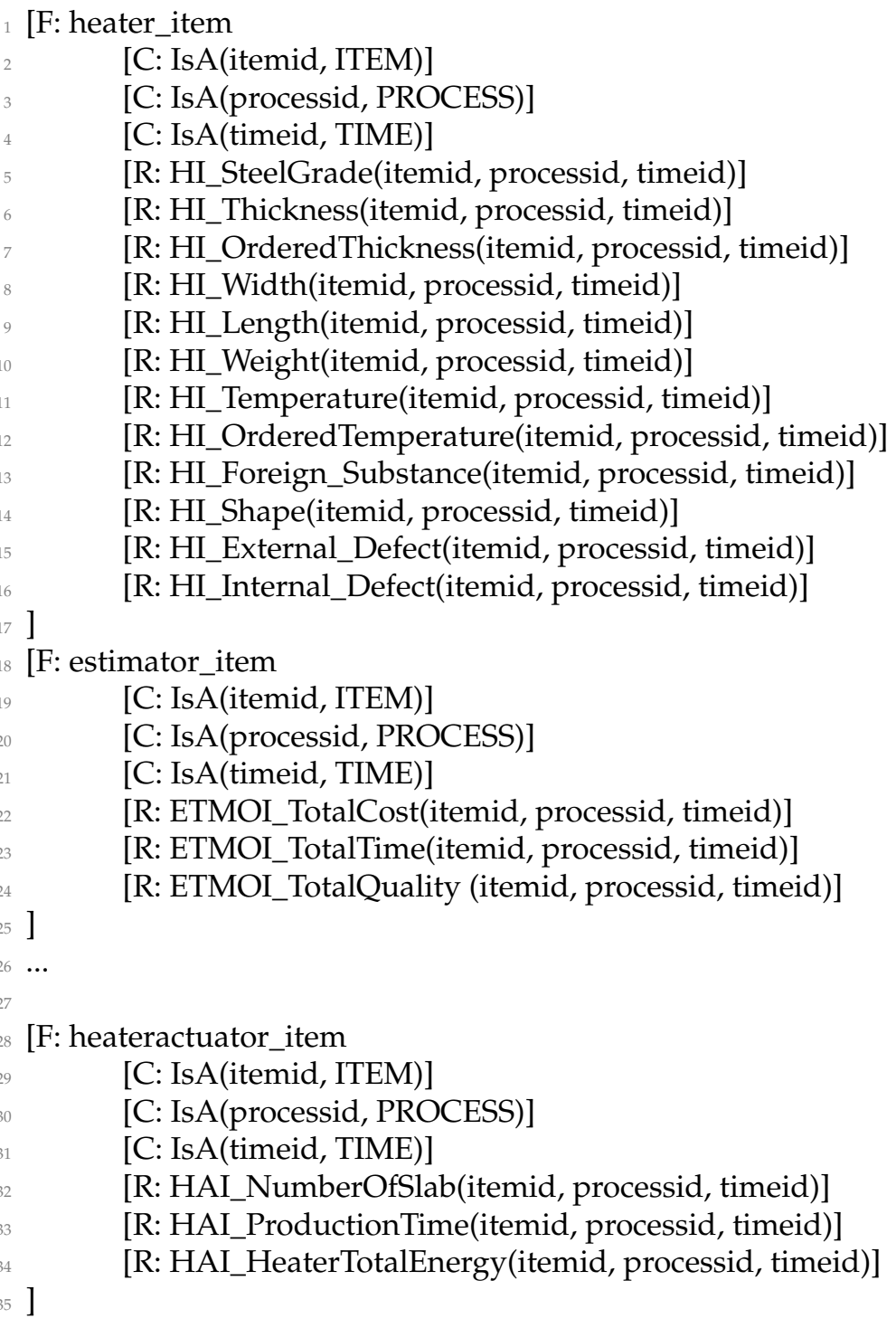




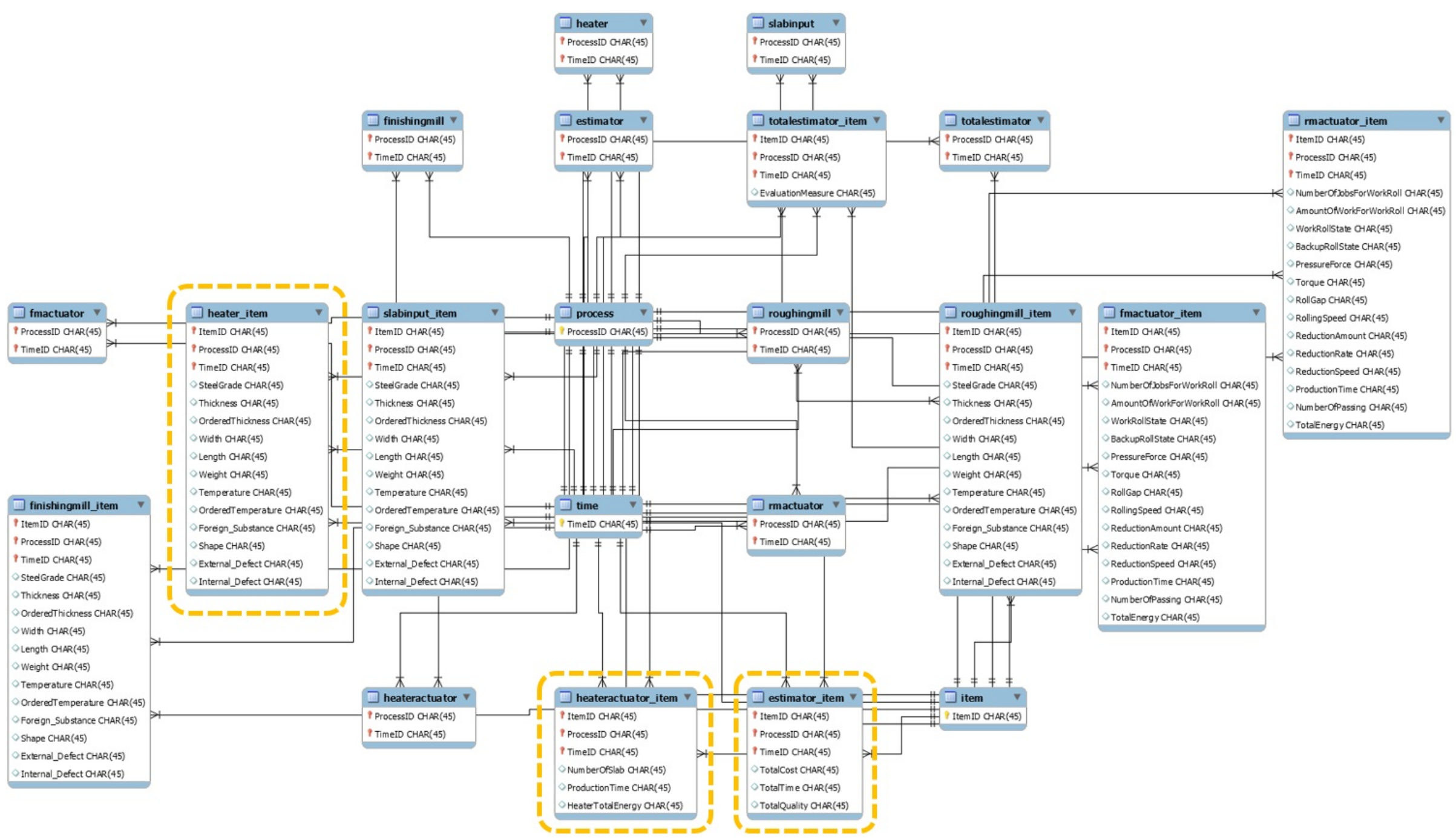

Figure 5. Partial MSAW Relational Database Schema. 
The MEBN-RM mapping for the partial MSAW relational database performed as we expected, and the total mapping time was $0.0161 \mathrm{~s}$ (Table 7).

Table 7. The Results of the MEBN-RM Mapping for the Partial MSAW Relational Database (Figure 5) and Its Mapping Time.

\begin{tabular}{ccccc}
\hline \# of Entity & $\begin{array}{c}\text { \# of MFrag } \\
\text { (Definition 1) }\end{array}$ & $\begin{array}{c}\text { \# of Resident Node } \\
\text { (Definition 2) }\end{array}$ & $\begin{array}{c}\text { \# of IsA Nodes } \\
\text { (Definition 4) }\end{array}$ & MappingTime (Second) \\
\hline 3 & 18 & 92 & 45 & 0.0161 \\
\hline
\end{tabular}

\section{Conclusions}

In this paper, we presented MEBN-RM formalizing conversion from a relational database schema in RM to a partial MTheory in MEBN syntactically. To do this, MEBN-RM contained the four levels of the mappings between elements of the relational database schema and MTheory. Table 8 summarizes the mappings that this research presents.

Table 8. Mapping types on MEBN-RM.

\begin{tabular}{|c|c|c|}
\hline RM & Mapping Types & MEBN \\
\hline ERS & $\begin{array}{l}\text { Definition } 18 \\
\text { ERS to Entity Mapping }\end{array}$ & Entity \\
\hline RRS & $\begin{array}{c}\text { Definition } 19 \\
\text { Predicate Resident Node Mapping }\end{array}$ & Predicate resident node \\
\hline $\begin{array}{l}\text { Non-foreign-key attribute, } \\
\text { Non-primary foreign key }\end{array}$ & $\begin{array}{c}\text { Definition } 20 \\
\text { Function Resident Node Mapping }\end{array}$ & Function resident node \\
\hline RS & $\begin{array}{c}\text { Definition } 21 \\
\text { RS-MFrag Mapping }\end{array}$ & MFrag \\
\hline RDBS & $\begin{array}{c}\text { Definition } 22 \\
\text { RDBS-MTheory Mapping }\end{array}$ & MTheory \\
\hline
\end{tabular}

MEBN-RM is a foundation of designing an MEBN model from a relational database, so, using MEBN-RM, the modeler (Human or Machine) can design the MEBN model seamlessly. The idea behind MEBN-RM may be used to develop other mapping models for different types of database (e.g., ontology, graph, and event database) as an example mapping model. Recently non-relational databases, called NoSQL, are receiving increasing attention. In the era of Big Data, we may need a scalable and flexible database to manage the many and varied types of data. In this paper, we only focused on the RM as a source data model to develop an MTheory. Future work will consider extensions to NoSQL data and other types of data.

Author Contributions: conceptualization, C.Y.P.; methodology, C.Y.P.; software, C.Y.P.; validation, C.Y.P. and K.B.L.; writing-original draft preparation, C.Y.P.; writing-review and editing, K.B.L.; supervision, K.B.L.; funding acquisition, K.B.L.

Funding: The research was partially supported by the Office of Naval Research (ONR), under Contract\#: N00173-09-C-4008.

Acknowledgments: We appreciate Paulo Costa and Shou Matsumoto for their helpful comments on this research. We also sincerely thank Shin at Samsung Thales for advice on the HERALD MTheory and simulation.

Conflicts of Interest: The authors declare no conflict of interest. 


\section{References}

1. Koller, D.; Friedman, N.; Džeroski, S.; Sutton, C.; McCallum, A.; Pfeffer, A.; Abbeel, P.; Wong, M.F.; Heckerman, D.; Meek, C.; et al. Introduction to Statistical Relational Learning; MIT Press: Cambridge, MA, USA, 2007.

2. MüUller, W.; Kuwertz, A.; Mühlenberg, D.; Sander, J. Semantic information fusion to enhance situational awareness in surveillance scenarios. In Proceedings of the 2017 IEEE International Conference on Multisensor Fusion and Integration for Intelligent Systems (MFI), Daegu, Korea, 16-18 November 2017; pp. 397-402.

3. Morariu, V.I.; Davis, L.S. Multi-agent event recognition in structured scenarios. In Proceedings of the CVPR 2011, Colorado Springs, CO, USA, 20-25 June 2011; pp. 3289-3296.

4. Wu, C.; Aghajan, H. User-centric environment discovery with camera networks in smart homes. IEEE Trans. Syst. Man Cybern. Part A Syst. Hum. 2011, 41, 375-383. [CrossRef]

5. Lippi, M.; Frasconi, P. Prediction of protein $\beta$-residue contacts by Markov logic networks with grounding-specific weights. Bioinformatics 2009, 25, 2326-2333. [CrossRef] [PubMed]

6. Poole, D. Probabilistic Horn abduction and Bayesian networks. Artif. Intell. 1993, 64, 81-129. [CrossRef]

7. Sato, T.; Kameya, Y. PRISM: A language for symbolic-statistical modeling. In Proceedings of the Fifteenth International Joint Conference on Artificial Intelligence (IJCAI 97), Nagoya, Japan, $23-29$ August 1997; Volume 97, pp. 1330-1339.

8. Koller, D. Probabilistic relational models. In Proceedings of the International Conference on Inductive Logic Programming, Bled, Slovenia, 24-27 June 1999; pp. 3-13.

9. Jaeger, M. Relational bayesian networks. In Proceedings of the Thirteenth Conference on Uncertainty in Artificial Intelligence, Providence, RI, USA, 1-3 August 1997; pp. 266-273.

10. Taskar, B.; Abbeel, P.; Koller, D. Discriminative probabilistic models for relational data. In Proceedings of the Eighteenth Conference on Uncertainty in Artificial Intelligence, Edmonton, AB, Canada, 1-4 August 2002; pp. 485-492.

11. Milch, B.; Marthi, B.; Russell, S.; Sontag, D.; Ong, D.L.; Kolobov, A. BLOG: Probabilistic models with unknown objects. In Proceedings of the IJCAI International Joint Conference on Artificial Intelligence, Edinburgh, UK, 30 July-5 August 2005; pp. 1352-1359.

12. Richardson, M.; Domingos, P. Markov logic networks. Mach. Learn. 2006, 62, 107-136. [CrossRef]

13. Gutmann, B.; Kersting, K. TildeCRF: Conditional random fields for logical sequences. In Proceedings of the European Conference on Machine Learning, Berlin, Germany, 18-22 September 2006; pp. 174-185.

14. Kersting, K.; De Raedt, L. Bayesian Logic Programming: Theory and Tool. In Introduction to Statistical Relational Learning; MIT Press: Cambridge, MA, USA, 2007; 291p.

15. McCallum, A.; Schultz, K.; Singh, S. Factorie: Probabilistic programming via imperatively defined factor graphs. In Advances in Neural Information Processing Systems; Curran Associates Inc.: Vancouver, BC, Canada, 2009; pp. 1249-1257.

16. Beierle, C.; Finthammer, M.; Potyka, N.; Varghese, J.; Kern-Isberner, G. A Framework for Versatile Knowledge and Belief Management Operations in a Probabilistic Conditional Logic. J. Log. 2017, 4, 2063-2095.

17. Pearl, J. Probabilistic Reasoning in Intelligent Systems: Networks of Plausible Inference; Morgan Kaufmann: San Francisco, CA, USA, 1988.

18. Laskey, K.B.; D'ambrosio, B.; Levitt, T.S.; Mahoney, S. Limited rationality in action: Decision Support for military situation assessment. Minds Mach. 2000, 10, 53-77. [CrossRef]

19. Wright, E.; Mahoney, S.; Laskey, K.; Takikawa, M.; Levitt, T. Multi-entity Bayesian networks for situation assessment. In Proceedings of the Fifth International Conference on Information Fusion, Annapolis, MD, USA, 8-11 July 2002; Volume 2, pp. 804-811.

20. Suzic, R. A generic model of tactical plan recognition for threat assessment. In Multisensor, Multisource Information Fusion: Architectures, Algorithms, and Applications 2005; International Society for Optics and Photonics: Orlando, FL, USA, 2005; Volume 5813, pp. 105-117.

21. Costa, P.C.G.; Carvalho, R.N.; Laskey, K.B.; Park, C.Y. Evaluating uncertainty representation and reasoning in HLF systems. In Proceedings of the 14th International Conference on Information Fusion, Chicago, IL, USA, 5-8 July 2011; pp. 1-8. 
22. Costa, P.C.G.; Laskey, K.B.; Chang, K.C.; Sun, W.; Park, C.Y.; Matsumoto, S. High-level information fusion with bayesian semantics. In Proceedings of the 9th Bayesian Modelling Applications Workshop, Catalina Island, CA, USA, 18 August 2012.

23. Park, C.Y.; Laskey, K.B.; Costa, P.C.G.; Matsumoto, S. Predictive situation awareness reference model using multi-entity bayesian networks. In Proceedings of the 17th International Conference on Information Fusion (FUSION), Salamanca, Spain, 7-10 July 2014; pp. 1-8.

24. Park, C.Y.; Laskey, K.B.; Costa, P.C.G. An Extended Maritime Domain Awareness Probabilistic Ontology Derived from Human-aided Multi-Entity Bayesian Networks Learning. In Proceedings of the Eleventh Conference on Semantic Technology for Intelligence, Defense, and Security, STIDS 2016; Fairfax, VA, USA, 14-17 November 2016; pp. 28-36.

25. Golestan, K. Information Fusion Methodology for Enhancing Situation Awareness in Connected Cars Environment. Ph.D. Dissertation, University of Waterloo, Waterloo, ON, Canada, 2015.

26. Li, X.; Martínez, J.F.; Rubio, G. Towards a hybrid approach to context reasoning for underwater robots. Appl. Sci. 2017, 7, 183. [CrossRef]

27. Park, C.Y.; Laskey, K.B.; Salim, S.; Lee, J.Y. Predictive situation awareness model for smart manufacturing. In Proceedings of the 2017 20th International Conference on Information Fusion (Fusion), Xi'an, China, 10-13 July 2017; pp. 1-8.

28. Golestan, K.; Soua, R.; Karray, F.; Kamel, M.S. Situation awareness within the context of connected cars: A comprehensive review and recent trends. Inf. Fusion 2016, 29, 68-83. [CrossRef]

29. Baum, L.E.; Petrie, T. Statistical inference for probabilistic functions of finite state Markov chains. Ann. Math. Stat. 1966, 37, 1554-1563. [CrossRef]

30. Hopfield, J.J. Artificial neural networks. IEEE Circuits Devices Mag. 1988, 4, 3-10. [CrossRef]

31. Cortes, C.; Vapnik, V. Support-vector networks. Mach. Learn. 1995, 20, 273-297. [CrossRef]

32. Pan, H.; Liu, L. Fuzzy bayesian networks-A general formalism for representation, inference and learning with hybrid bayesian networks. Int. J. Pattern Recognit. Artif. Intell. 2000, 14, 941-962. [CrossRef]

33. Murphy, K.P.; Russell, S. Dynamic Bayesian Networks: Representation, Inference and Learning. Ph.D. Dissertation, University of California, Berkeley, CA, USA, 2002.

34. Patnaikuni, P.; Shrinivasan, R.; Gengaje, S.R. Survey of Multi Entity Bayesian Networks (MEBN) and its applications in probabilistic reasoning. Int. J. Adv. Res. Comput. Sci. 2017, 8, 2425-2429.

35. Costa, P.C.G. Bayesian Semantics for the Semantic Web. Ph.D. Dissertation, George Mason University, Fairfax, VA, USA, 2005.

36. Carvalho, R.N.; Laskey, K.B.; Costa, P.C.G. PR-OWL-A language for defining probabilistic ontologies. Int. J. Approx. Reason. 2017, 91, 56-79. [CrossRef]

37. Carvalho, R.N.; Laskey, K.B.; Da Costa, P.C.G. Uncertainty modeling process for semantic technology. Peerj Comput. Sci. 2016, 2, e77. [CrossRef]

38. Gershenfeld, N.; Krikorian, R.; Cohen, D. The internet of things. Sci. Am. 2004, 291, 76-81. [CrossRef] [PubMed]

39. Guinard, D.; Trifa, V. Towards the web of things: Web mashups for embedded devices. In Proceedings of the WWW (International World Wide Web Conferences), Madrid, Spain, 20-24 April 2009; Volume 15.

40. Sekkal, N.; Benslimane, S.M.; Mrissa, M.; Park, C.Y.; Boudaa, B. Proactive and reactive context reasoning architecture for smart web services. Int. J. Data Min. Model. Manag. 2019, in press.

41. Codd, E.F. A relational model of data for large shared data banks. Commun. ACM 1970, 13, 377-387. [CrossRef]

42. Codd, E.F. Further normalization of the data base relational model. Data Base Syst. 1972, RJ909, 33-64.

43. Codd, E.F. Racent Investigations in Relational Data Base Systems. Inf. Process. 1974, RJ1385, 1017-1021.

44. Han, J.; Haihong, E.; Le, G.; Du, J. Survey on NoSQL database. In Proceedings of the 2011 6th International Conference on Pervasive Computing And Applications, Port Elizabeth, South Africa, 26-28 October 2011; pp. 363-366.

45. Park, C.Y.; Laskey, K.B.; Costa, P.C.G.; Matsumoto, S. A process for human-aided Multi-Entity Bayesian Networks learning in Predictive Situation Awareness. In Proceedings of the 2016 19th International Conference on Information Fusion (FUSION), Heidelberg, Germany, 5-8 July 2016; pp. 2116-2124. 
46. Park, C.Y.; Laskey, K.B.; Costa, P.C.G.; Matsumoto, S. Multi-entity bayesian networks learning for hybrid variables in situation awareness. In Proceedings of the 16th International Conference on Information Fusion, Istanbul, Turkey, 9-12 July 2013; pp. 1894-1901.

47. Park, C.Y.; Laskey, K.B.; Costa, P.C.G.; Matsumoto, S. Multi-Entity Bayesian Networks Learning in Predictive Situation Awareness. In Proceedings of the 18th International Command and Control Technology and Research Symposium, Alexandria, VA, USA, 19-21 June 2013.

48. Laskey, K.B. MEBN: A language for first-order Bayesian knowledge bases. Artif. Intell. 2008, 172, 140-178. [CrossRef]

49. Fagin, R. The decomposition versus synthetic approach to relational database design. In Proceedings of the Third International Conference on Very Large Data Bases, Tokyo, Japan, 6-8 October 1977; Volume 3, pp. 441-446.

50. Fagin, R. Normal forms and relational database operators. In Proceedings of the 1979 ACM SIGMOD International Conference on Management of Data, Boston, MA, USA, 30 May-1 June 1979; pp. 153-160.

51. Maier, D. Theory of Relational Databases; Computer Science Pr: Rockville, MD, USA, 1983.

52. Date, C. Database Design and Relational Theory: Normal Forms and All That Jazz; O'Reilly Media, Inc.: Sebastopol, CA, USA, 2012.

53. Motl, J.; Schulte, O. The CTU prague relational learning repository. arXiv 2015, arXiv:1511.03086.

(C) 2019 by the authors. Licensee MDPI, Basel, Switzerland. This article is an open access article distributed under the terms and conditions of the Creative Commons Attribution (CC BY) license (http:/ / creativecommons.org/licenses/by/4.0/). 